\title{
Simulation of the microwave emission of multi-layered snowpacks using the Dense Media Radiative transfer theory: the DMRT-ML model
}

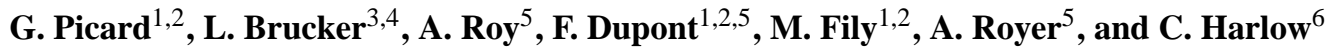 \\ ${ }^{1}$ CNRS, LGGE UMR5183, 38041 Grenoble, France \\ ${ }^{2}$ Univ. Grenoble Alpes, LGGE (UMR5183), 38041 Grenoble, France \\ ${ }^{3}$ NASA Goddard Space Flight Center, Cryospheric Sciences Lab., code 615 Greenbelt, MD, 20771 USA \\ ${ }^{4}$ Goddard Earth Sciences Technology and Research Studies and Investigations, Universities Space Research Association, \\ Greenbelt, MD, 20771 USA \\ ${ }^{5}$ Centre d'applications et de recherches en télédétection (CARTEL), Université de Sherbrooke, 2500 Bd Université, \\ Sherbrooke, QC J1K 2R1 Canada \\ ${ }^{6}$ Met Office, EX1 3PB Exeter, UK
}

Correspondence to: G. Picard (ghislain.picard@ujf-grenoble.fr)

Received: 20 September 2012 - Published in Geosci. Model Dev. Discuss.: 14 November 2012

Revised: 23 May 2013 - Accepted: 7 June 2013 - Published: 26 July 2013

\begin{abstract}
DMRT-ML is a physically based numerical model designed to compute the thermal microwave emission of a given snowpack. Its main application is the simulation of brightness temperatures at frequencies in the range $1-200 \mathrm{GHz}$ similar to those acquired routinely by spacebased microwave radiometers. The model is based on the Dense Media Radiative Transfer (DMRT) theory for the computation of the snow scattering and extinction coefficients and on the Discrete Ordinate Method (DISORT) to numerically solve the radiative transfer equation. The snowpack is modeled as a stack of multiple horizontal snow layers and an optional underlying interface representing the soil or the bottom ice. The model handles both dry and wet snow conditions. Such a general design allows the model to account for a wide range of snow conditions. Hitherto, the model has been used to simulate the thermal emission of the deep firn on ice sheets, shallow snowpacks overlying soil in Arctic and Alpine regions, and overlying ice on the large icesheet margins and glaciers. DMRT-ML has thus been validated in three very different conditions: Antarctica, Barnes Ice Cap (Canada) and Canadian tundra. It has been recently used in conjunction with inverse methods to retrieve snow grain size from remote sensing data. The model is written in Fortran90 and available to the snow remote sensing community as an open-source software. A convenient user interface is provided in Python.
\end{abstract}

\section{Introduction}

Passive microwave radiometers on-board satellites acquire useful observations for the characterization of snow-covered areas. These observations are available in these areas several times a day, are relatively insensitive to the atmosphere in many frequency bands, and are independent of the solar illumination. They are sensitive to several properties relevant for monitoring the snow cover and have been exploited in numerous algorithms to retrieve continental snow cover extent (Grody and Basist, 1996), snow depth and snow water equivalent on both land (Josberger and Mognard, 2002; Kelly and Chang, 2003; Derksen et al., 2003) and sea ice (Cavalieri et al., 2012; Brucker and Markus, 2013), snow accumulation on ice sheets (Abdalati and Steffen, 1998; Vaughan et al., 1999; Winebrenner et al., 2001; Arthern et al., 2006), melt events (Abdalati and Steffen, 1997; Picard and Fily, 2006), snow temperature (Shuman et al., 1995; Schneider, 2002; Schneider et al., 2004), and snow grain size (Brucker et al., 2010; Picard et al., 2012). Some of these studies are based on empirical relationships supported by physical interpretations (Koenig et al., 2007) and others directly use physical models and data assimilation techniques (Durand and Margulis, 2007; Picard et al., 2009; Takala et al., 2011; Toure et al., 2011; Huang et al., 2012). In both cases, understanding 
and modeling the physical processes of the microwave emission by snow and the underlying surface are crucial.

Modeling the snow microwave emission from snow physical properties takes in general two successive steps. The first step is the computation of the electromagnetic properties (e.g., effective dielectric constant, scattering and absorption coefficients) that characterize the propagation and the single events of interaction between wave and matter. These properties are calculated from the geometric micro-structural properties of the snow, assumed to be homogeneous within a given layer. The second step is the computation of the emission and the propagation through the snowpack by accounting for the multiple interactions of microwaves within the snow as well as by refraction, reflection and transmission at the interfaces. It is often treated with the radiative transfer equation on a plane-parallel medium for which generic solutions exist (Tsang et al., 1985; Fung, 1994). In contrast, the first step is specific to the medium. In the case of snow, the main challenge for the electromagnetic calculation is the high density of scatterers. The volume occupied by the scatterers over the total volume (referred to as the fractional volume $f$ ) is significant - typically larger than $20 \%$-, which implies that the scatterers strongly interact with each other and the independent scattering theory used for vegetation or clouds (Tsang et al., 1985; Ulaby et al., 1986; Chuah and Tan, 1989) is inadequate. Several empirical formulas relating the scattering and absorption coefficients to grain size and density have been proposed to solve this issue and are found in the Helsinki University of Technology model (HUT/TKK) (Pulliainen et al., 1999; Lemmetyinen et al., 2010) and in the Microwave Emission Model of Layered Snowpacks (MEMLS) (Wiesmann and Mätzler, 1999). The derivation of relationships from Maxwell's equations is an attractive alternative because it is independent of particular snow conditions and the assumptions are explicit:

- the strong fluctuation theory (SFT, Stogryn, 1986) allows calculations at low frequencies typically less than $20 \mathrm{GHz}$;

- to cover the frequency range of $1-100 \mathrm{GHz}$, which is relevant for existing radiometers, Mätzler (1998) proposed the improved Born approximation (IBA). As in the SFT, the size of grains is given by the correlation length (Mätzler, 2002). This metric is well defined but direct measurements are only possible from 2-D or 3D micro-structure data, which require advanced experimental techniques (Wiesmann et al., 1998). Estimations can be indirectly obtained from quantities measurable in the field like the snow specific surface area (Mätzler, 2002; Arnaud et al., 2011) or the micro-penetration profile (Löwe and van Herwijnen, 2012);

- the dense media radiative transfer theory (DMRT; Tsang et al., 1985; West et al., 1993; Shih et al., 1997; Tsang et al., 2000a, 2007) considers snow as a collec- tion of spherical ice particles and provides analytical formulas of the effective propagation wave number, the scattering and absorption coefficients as a function of the sphere radius and the density. It is attractive because sphere radius and snow density are well defined and, when the snow grains are close to spheres like in fine grained snow (Colbeck, 1993), they are both measurable in the field. Moreover, the DMRT-ML theory has solid theoretical grounds and has been regularly improved during the last two decades.

These theories have been implemented in several models: the SFT in Surdyk and Fily (1995), the IBA in MEMLS (Mätzler and Wiesmann, 1999) and the DMRT-ML in Macelloni et al. (2001); Tedesco and Kim (2006); Liang et al. (2008); Grody (2008); Brogioni et al. (2009). In addition to the underlying theory, the models differ by many aspects, including the methods for solving the radiative transfer, the presence of smooth or rough interfaces, the possible number of layers, etc. In particular, HUT uses the two-stream method and MEMLS the six-stream method while most DMRT-based models consider a larger number of streams. The comparison for a large variety of snow types (Tedesco and Kim, 2006) showed that no particular model systematically reproduces all of the experimental data. It is not yet known if the discrepancies were attributable to the electromagnetic theory, specific details of the implementations of the models, or uncertainties pertaining to input or evaluation data. Moreover, the different representations of the snow grain size in these different approaches are a major limit to the comparison. Both MEMLS and HUT/TKK models are widely used (Butt and Kelly, 2008; Durand et al., 2008; Rees et al., 2010; Brucker et al., 2011b; Gunn et al., 2011). In contrast, several groups use home-made DMRT-based models but no widely-used reference implementation exists, which limits the spread of this theory and limits the comparisons between studies.

The objective of this paper is to present the DMRT-ML (DMRT Multi-Layer) model that was released under an open source license and accompanied by a detailed documentation (http://lgge.osug.fr/ picard/dmrtml/). This model initially developed at Laboratoire de Glaciologie and Géophysique de l'Environment in Grenoble, France, for perennial snowpack (Brucker et al., 2010, 2011a) and improved for seasonal snow on soil (Roy et al., 2013) and on superimposed ice (Dupont et al., 2012), is now suitable for modeling the microwave emission in a wide range of snowy continental environments. It is also designed to be extensible and uses standard Fortran90, which allows efficient computations with different types of computers and operating systems and facilitates the embedding in other models or assimilation schemes. A convenient user interface is provided by the optional bindings with the Python language. 
This paper is a comprehensive scientific reference for DMRT-ML users. It is structured as follows. Section 2 describes DMRT-ML in detail. Section 3 presents the sensitivity to the most important input variables and parameters and provides practical recommendations on the validity range of the input variables and parameters to use. Section 4 summarizes the results of the detailed comparisons (Brucker et al., 2011a; Roy et al., 2013; Dupont et al., 2012) with radiometric data obtained for various cold environments.

\section{The DMRT-ML model}

\subsection{Radiative transfer equation and model architecture}

The energy emanating from snowpacks is the result of the thermal emission of the snow, the substratum and the atmosphere, and of the complex propagation of this energy toward the upper snow layers. The emission and propagation can be described by the radiative transfer equation. Assuming that the medium is a stack of $L$ plane-parallel layers containing an isotropic and homogeneous material, the equation in every layer is (Jin, 1994)

$$
\begin{aligned}
& \cos \theta \frac{\mathrm{d}}{\mathrm{d} z} \boldsymbol{T}_{\mathrm{B}}(z, \theta, \phi)=-\kappa_{\mathrm{e}} \boldsymbol{T}_{\mathrm{B}}(z, \theta, \phi) \\
& +\int_{0}^{\pi / 2} \int_{0}^{2 \pi} \sin \theta^{\prime} \mathrm{d} \theta^{\prime} \mathrm{d} \phi^{\prime} \mathbf{P}\left(\theta, \phi, \theta^{\prime}, \phi^{\prime}\right) \boldsymbol{T}_{\mathrm{B}}\left(z, \theta^{\prime}, \phi^{\prime}\right)+\kappa_{\mathrm{a}} T(z) \boldsymbol{I} .
\end{aligned}
$$

By convention, matrices are written in bold, and vectors in bold and italic. $\boldsymbol{I}$ is the unit column vector. $\boldsymbol{T}_{\mathrm{B}}(z, \theta, \phi)$ is the radiance at depth $z$ propagating along the direction with zenith angle $\theta$ and azimuth angle $\phi$. According to the Rayleigh Jeans approximation, that is valid in the microwave range, $\boldsymbol{T}_{\mathrm{B}}(z, \theta, \phi)$ also represents the brightness temperature. Since the medium is isotropic, $\boldsymbol{T}_{\mathrm{B}}$ is a vector with only two non-null components (Jin, 1994, p. 19): the vertically and horizontally polarized brightness temperature. $\kappa_{\mathrm{e}}$ and $\kappa_{\mathrm{a}}$ are the extinction and absorption coefficients in the layer and $\mathbf{P}$ is the phase function. $T$ is the physical temperature of the layer.

The model solves this equation for the particular medium described by the input variables and parameters (Fig. 1). Several steps are needed: the determination of the dielectric properties of the constitutive materials (Sect. 2.2), the determination of the effective propagation constant and of the extinction and scattering coefficients for each homogeneous layer of the medium (Sect. 2.3) by using the DMRT-ML theory, the determination of the boundary conditions (Sect. 2.4) and lastly the numerical computation of the solution of the radiative transfer equation (Sect. 2.5). The result is the brightness temperature emerging from the surface in several directions. Finally, other relevant variables are accessible via additional post-computations (Sect. 2.6).

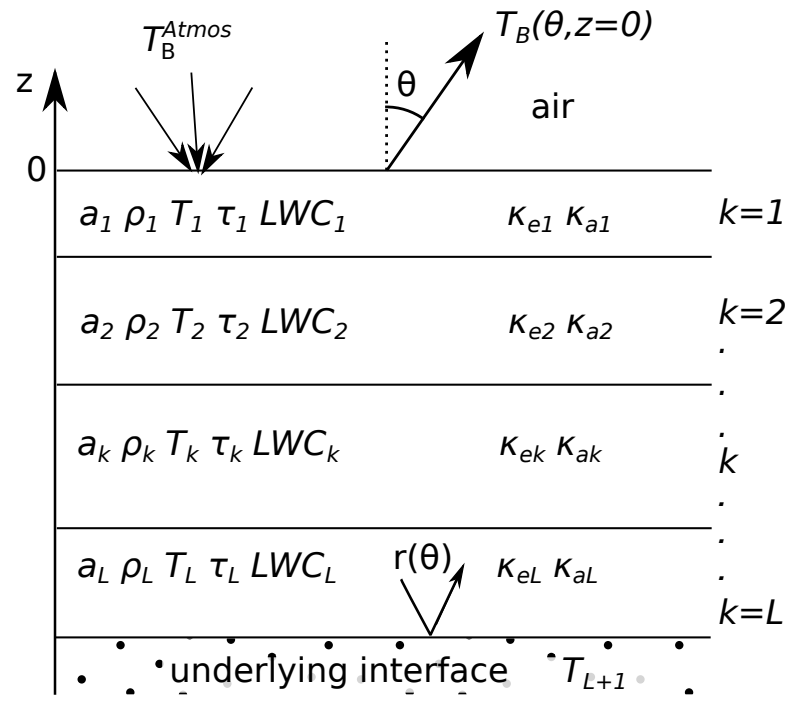

Fig. 1. The snowpack viewed by DMRT-ML. $L$ is the number of layers. For each homogeneous layer $k$, the input variables and parameters are grain size $a_{k}$, snow density $\rho_{k}$, physical temperature $T_{k}$, stickiness $\tau_{k}$ and liquid water content $\mathrm{LWC}_{k}$. The extinction and absorption coefficients $\kappa_{\mathrm{e}}$ and $\kappa_{\mathrm{a}}$ are calculated with the DMRTML theory. The boundary conditions require the input of the downwelling brightness temperature $T_{\mathrm{B}}^{\mathrm{atmos}}$ and the variables and parameters to compute the reflection coefficient $r(\theta)$ of the underlying interface (see Table 1). The DISORT method is used to solve the radiative transfer equation in the snowpack and provides as output the brightness temperature $T_{\mathrm{B}}(\theta, z=0)$ emerging from the snowpack.

\subsection{Dielectric constants}

The computation of the scattering and extinction coefficients in the DMRT-ML theory requires the dielectric constant of the scatterer and background materials. When snow is dry (temperature strictly below the melting point), the grains are assumed to be composed of pure ice, whose dielectric con-

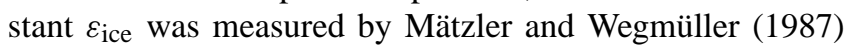
and Mätzler et al. (2006):

$$
\begin{aligned}
\varepsilon_{\text {ice }} & =3.1884+0.00091(T-273.0)+J\left(\frac{\alpha}{v}+\beta v\right), \\
\alpha & =(0.00504+0.0062 \Theta) \exp (-22.1 \Theta), \\
\beta & =\frac{0.0207}{T} \frac{\exp \left(\frac{335}{T}\right)}{\left(\exp \left(\frac{335}{T}\right)-1\right)^{2}} \\
& +1.1610^{-11} v^{2}+\exp (-9.963+0.0372(T-273.16)), \\
\Theta & =\frac{300}{T}-1,
\end{aligned}
$$

where $v$ is the frequency in $\mathrm{GHz}, T$ the ice temperature in $\mathrm{K}$ and $\mathrm{J}^{2}=-1$. This model is valid for temperatures above $240 \mathrm{~K}$ and in the microwave range $(1-200 \mathrm{GHz})$ but its accuracy certainly decreases at low frequencies (Jiang and $\mathrm{Wu}, 2004)$. 
When the snow is wet (at $273.15 \mathrm{~K}$ ), ice grains are coated by liquid water with an uneven distribution controlled by capillarity forces. Since such a complex particle cannot be taken explicitly into account in the DMRT-ML theory and given the low content of liquid water usually present in moist and wet snow (less than $8 \%$; Colbeck, 1993), DMRT-ML assumes that the grains are composed of a fictitious homogeneous material. The dielectric constant $\varepsilon_{\text {wet ice }}$ of this material is calculated using the mixture relationship (Borghese et al., 2007; Chopra and Reddy, 1986):

$$
\begin{aligned}
\varepsilon_{\text {wet ice }} & =\frac{C_{+}+2 C_{-}}{C_{+}-C_{-}} \varepsilon_{\text {water }}, \\
C_{+} & =\varepsilon_{\text {ice }}+\varepsilon_{\text {water }}, \\
C_{-} & =\left(\varepsilon_{\text {ice }}-\varepsilon_{\text {water }}\right)(1-\text { LWC }),
\end{aligned}
$$

where LWC is the liquid water content expressed here as the ratio between the volume of liquid water and the volume of ice present in snow (in $\mathrm{m}^{3} \mathrm{~m}^{-3}$ ) and the water dielectric constant $\varepsilon_{\text {water }}$ is given by

$$
\begin{aligned}
\varepsilon_{\text {water }} & =\varepsilon_{2}+\frac{\varepsilon_{1}-\varepsilon_{2}}{1-J \frac{v}{\nu_{2}}}+\frac{\varepsilon_{0}-\varepsilon_{1}}{1-J \frac{v}{v_{1}}}, \\
\varepsilon_{0} & =77.66+103.3 \Theta, \\
\varepsilon_{1} & =0.0671 \varepsilon_{0}, \\
\varepsilon_{2} & =3.52+7.52 \Theta, \\
\nu_{1} & =20.2-146.4 \Theta-316 \Theta^{2}, \\
\nu_{2} & =39.8 v_{1} .
\end{aligned}
$$

\subsection{Snow extinction and absorption coefficients, and phase function: the DMRT-ML theory}

The extinction and absorption coefficients, as well as the form of the phase function are obtained by the so-called DMRT-ML theory. Different versions of this theory have been published over time and the underlying assumptions can significantly differ from each other. Four characteristics distinguish these versions: (i) the underlying approximation used for the DMRT-ML derivation, (ii) the particle size with respect to the wavelength, (iii) the stickiness between particles and (iv) the distribution of particle size. DMRT-ML proposes some of these versions and allows various options as detailed here.

DMRT-ML uses the quasicrystalline approximation with coherent potential (QCA-CP) (Tsang et al., 2000b) and is limited to particle size smaller than the wavelength. This may be a limitation at frequencies higher than $37 \mathrm{GHz}$ and with large grains commonly found in aged snow. The calculation for large particles requires a Mie-like development (Tsang et al., 2000a) and is computationally much more intensive than the QCA-CP calculation. In addition, it leads to a form of the phase function that is incompatible with the optimization of the radiative transfer equation resolution used in DMRT-ML (Sect. 2.5). To avoid the strong divergence that characterized QCA-CP with large particles, Grody (2008) proposed an empirical and computationally efficient treatment of this issue. He noticed that snow is composed of particles with a broad range of sizes, which results in a smoothing of the undulation characteristic of the Mie resonances. Hence, a good approximation of the medium scattering efficiency $Q_{\text {s }}$ for large particles is the asymptotic limit, i.e., $Q_{\mathrm{s}} \approx 2$, accounting from the fact that the absorption is weak (Twomey and Bohren, 1980). If enabled by the user, DMRTML applies this idea by limiting the $Q_{\mathrm{s}}$ maximum value to 2 . Nevertheless, this ad hoc correction has not the objective to replace the rigorous solution for fine-grained studies (Tsang et al., 2000a).

Recent versions of DMRT-ML introduce the concept of stickiness. Instead of considering randomly positioned nonpenetrable spheres, the sticky spheres are attracted to one another and tend to form clusters with large voids between. This concept is meant to better represent the micro-structure of natural snow using solely spherical grains. In this case, the stickiness is also a means to account for coarse grained snow by considering that such snow is made of small clustered particles. However, DMRT-ML only implements the "short range" version of the sticky formulation, which assumes that the clusters are small with respect to the wavelength (Tsang et al., 2000b, pp. 504-505). In this simplified case, the phase function remains identical to that of the nonsticky small particle case for which the optimization of the resolution of the radiative transfer equation works (Sect. 2.5).

Another way to improve the representation of the microstructure of natural snow is to consider a collection of spheres with different sizes. In DMRT-ML, the particle sizes follow the Rayleigh distribution (Jin, 1994). In addition, only the nonsticky particle case is available because the formulation with both stickiness and size distribution leads to a quadratic system of equations that is difficult to solve and is computationally intensive (Tsang and Kong, 2001, p. 430).

In summary, DMRT-ML includes two implementations:

- QCA-CP mono-disperse, with optional "short range" stickiness, and optional Grody's-based empirical treatment of large particles;

- QCA-CP poly-disperse with a Rayleigh distribution, no stickiness and no large particles.

The mono-disperse version is formulated according to Shih et al. (1997). The effective dielectric constant without scattering $E_{\text {effo }}$ is obtained by solving the following quadratic Eq. (3) in Shih et al. (1997) with $a=0$ or Eq. (5.3.125) in Tsang and Kong (2001):

$$
E_{\text {effo }}^{2}+E_{\text {effo }}\left(\frac{\epsilon_{\mathrm{s}}-\epsilon_{\mathrm{b}}}{3}(1-4 f)-\epsilon_{\mathrm{b}}\right)-\epsilon_{\mathrm{b}} \frac{\epsilon_{\mathrm{s}}-1}{3}(1-f)=0,
$$

where $f$ is the fractional volume of scatterers, $\epsilon_{\mathrm{b}}$ and $\epsilon_{\mathrm{s}}$ are the dielectric constants of the background and scatterers, respectively. The effective dielectric constant with scattering 
(Eq. 3 in Shih et al., 1997) combined with Eq. (5) yields:

$$
\begin{aligned}
& E_{\text {eff }}=\varepsilon_{\mathrm{b}}+\left(E_{\text {eff } 0}-\varepsilon_{\mathrm{b}}\right) \\
& \left(1+J \frac{2}{9}\left(k_{0} a\right)^{3} \sqrt{E_{\text {eff } 0}} \frac{\varepsilon_{\mathrm{s}}-\varepsilon_{\mathrm{b}}}{1}\right. \\
& \left.+\frac{\varepsilon_{\mathrm{s}}-\varepsilon_{\mathrm{b}}}{3 E_{\text {effo }}}(1-f) \frac{(1-f)^{4}}{(1+2 f-t f(1-f))^{2}}\right),
\end{aligned}
$$

where $a$ is the radius of the spheres and $k_{0}=\pi / \lambda$ is the wave number with $\lambda$ the wavelength. $t$ is zero for nonsticky spheres and otherwise is the largest solution of Eq. 6 in Shih et al. (1997)

$$
\frac{f}{12} t^{2}-\left(\tau+\frac{f}{1-f}\right) t+\frac{1+f / 2}{(1-f)^{2}}=0,
$$

where $\tau$ is the stickiness parameter (Shih et al., 1997; Tsang and Kong, 2001, p. 430). At last, the extinction and absorption coefficients are respectively given by

$$
\begin{aligned}
& \kappa_{e}=2 k_{0} \Im \sqrt{E_{\text {eff }},} \\
& \kappa_{a}=\kappa_{e}-\kappa_{\mathrm{s}} \\
& \kappa_{\mathrm{s}}=\frac{2}{9} k_{0}^{4} a^{3} f\left|\frac{\epsilon_{\mathrm{s}}-\epsilon_{\mathrm{b}}}{1+\frac{\epsilon_{\mathrm{s}}-\epsilon_{\mathrm{b}}}{3 E_{\mathrm{eff}}}(1-f)}\right|^{2} \frac{(1-f)^{4}}{(1+2 f-t f(1-f))^{2}},
\end{aligned}
$$

with $\Im$ the imaginary part indicator and $\kappa_{\mathrm{S}}$ the scattering coefficient.

In the case of small particles, the phase function in DMRT-ML has the same form as with independent particles Rayleigh phase function (Jin, 1994, Eqs. 2-26 to 2-28).

The DMRT-ML theory inherits its name from the fact that it extends the classical radiative transfer theory, which requires the particles not to interact with each other. This condition is met only when the fractional volume $f$ is less than a few percent (Ishimaru and Kuga, 1982). Despite its name, recent works suggest the DMRT-ML theory does not work for any large fractional volumes (see Sect. 3.3 for details). As a consequence, for icy layers or layers subject to several melt-refreeze cycles, it is recommended to use the DMRTML theory for a collection of air bubbles embedded in ice background. This is achieved in DMRT-ML by exchanging the dielectric constant of ice and air in Eqs. (5) and (6).

\subsection{Properties of the interfaces}

Once the extinction and absorption coefficients and the phase function within every layer are determined, all the variables and parameters in Eq. (1) are known and a general solution can be obtained independently for every layer. To obtain the particular solution and hence the brightness temperature emerging from the surface, it is necessary to propagate the radiation between the individual snow layers. This propagation must ensure the energy conservation and requires the reflection properties at every interface as well as any external source of energy.
For the interfaces within the snowpack and at the airsnow interface, DMRT-ML considers smooth interfaces. The roughness of this interface due to ripples, sastrugi and other dunes (Watanabe, 1978) may contribute especially at horizontal polarization and grazing incidence angles (Lacroix et al., 2009), but the existing formulations of the bi-static reflection coefficient are based on the method of moments and are therefore computationally intensive and limited to low frequencies ( $<18 \mathrm{GHz}$; Liang et al., 2009; Chang and Tsang, 2011). For flat interfaces, the reflection coefficient only depends on the zenith angle and the refractive indexes of both sides of the interface. In DMRT-ML, it is obtained by Fresnel's relationships (e.g. Jin, 1994, p. 59) using the effective dielectric constants calculated with Eq. (6).

At the air-snow interface, the energy coming down from the atmosphere $T_{\mathrm{B}}^{\mathrm{atmos}}$ is an input variable given by the user. It is assumed isotropic in the current version of DRMT-ML but this assumption can be easily changed. The calculation of $T_{\mathrm{B}}^{\mathrm{atmos}}$ from meteorological data requires an external model such as proposed by Rosenkranz (1998) and Saunders et al. (1999).

At the bottom interface, to account for the diversity of media that can be present below snow covers (e.g., soil, ice, lake ice, sea ice) and the diversity of electromagnetic modeling approaches for the soil, several substratum models are available in DMRT-ML and the addition of new models is easy. The role of the substratum model is to provide the reflection coefficient of the interface. DMRT-ML only takes into account the reflection of the coherent wave and neglects the diffuse reflections at the bottom interface. It means that the roughness of the interface is only partially taken into account. In addition to reflecting downwelling energy, the substratum is an emitter: the energy emitted and entering into the snowpack is calculated using the temperature of the substratum given as an input variable and the emissivity deduced from the reflection coefficient and Kirchhoff's law.

DMRT-ML proposes 11 models that cover soil, ice, lake and semi-infinite snowpack. The user selects the type of substratum model using an identification number and provides the required variables and parameters depending on the type of model (Table 1). Since these substratum models have been published elsewhere, their evaluation is not addressed in this paper. In the case where snow lies over ice, DMRT-ML offers two options. If the ice is free of bubbles, isothermal and semiinfinite, the best option to use is the "ice" substratum model (ID 202, or ID 1 with ice dielectric constant). In any other conditions, it is recommended to represent the underlying ice using layers with a high density (up to $917 \mathrm{~kg} \mathrm{~m}^{-3}$ for bubble-free ice) and no substratum. The number of layers to use depends on the temperature and density profile in the ice. The total depth of the ice layers must be large enough to avoid "leakage" at the bottom. Special attention is required at low frequencies (like $\mathrm{L}$ band, $1.4 \mathrm{GHz}$ ) since ice absorption can be very weak and the layers well below $100 \mathrm{~m}$ can significantly contribute. 
Table 1. Available models for the reflection coefficient of the bottom interface and required input variables and parameters. SM is the soil moisture (volume fraction), $\varepsilon$ is the complex dielectric constant, $\sigma$ is the surface root mean square height (in meters), $f_{\text {clay }}$ and $f_{\text {sand }}$ are the fractions of clay and sand, $\rho_{\text {orga }}$ is the density of dry organic matter $\left(\mathrm{kg} \mathrm{m}^{-3}\right), T$ is the temperature (in $\left.\mathrm{K}\right)$ and $\mathrm{Q}$ and $\mathrm{H}$ are dimensionless parameters. P99 stands for Pulliainen et al. (1999), D85 for Dobson et al. (1985), M87 for Mätzler and Wegmüller (1987) and M06 for Mätzler et al. (2006).

\begin{tabular}{lcccr}
\hline Interface model & Material & Dielectric constant & ID & Variables and parameters \\
\hline No interface & None & & 0 & \\
Flat surface, Fresnel & Any & prescribed & 1 & \\
Flat surface, Fresnel & Soil & from P99 & 2 & $\mathrm{SM}, f_{\text {clay }}, f_{\text {sand }}, \rho_{\text {orga }}, T$ \\
Flat surface, Fresnel & Soil & from D85 & 3 & SM, $T$ \\
Rough surface WM99 & Any & prescribed & 101 & $\sigma, \varepsilon$ \\
Rough surface WM99 & Soil & from P99 & 102 & $\sigma, \mathrm{SM}, f_{\text {clay }}, f_{\text {sand }}, \rho_{\text {orga }}, T$ \\
Rough surface WM99 & Soil & from D85 & 103 & $\sigma, \mathrm{SM}, T$ \\
Flat surface, Fresnel & Ice & from M87, M06 & 202 & $T$ \\
QH model W83 & Any & prescribed & 301 & $\sigma, \mathrm{Q}, \mathrm{H}, \varepsilon$ \\
QH model W83 & Soil & from P99 & 302 & $\sigma, \mathrm{Q}, \mathrm{H}, \mathrm{SM}, f_{\text {clay }}, f_{\text {sand }}, \rho_{\text {orga }}, T$ \\
QH model W83 & Soil & from D85 & 303 & $\sigma, \mathrm{Q}, \mathrm{H}, \mathrm{SM}, T$ \\
Flat surface, Fresnel & Fresh water & from M87 & 402 & $T$ \\
\hline
\end{tabular}

\subsection{Solution of the radiative transfer equation using the DISORT method}

Once the extinction and scattering coefficients of every layer and the reflection coefficients at all the interfaces are known, the radiative transfer equation is solved using the DISORT method (Chandrasekhar, 1960). This method takes into account multiple scattering within and between the layers, which is an asset with respect to the iterative method (Tsang et al., 1985; Jin, 1994; Ishimaru, 1997) for which the number of calculated order of scattering is limited. It also computes the energy propagation in an unlimited number of directions (or "streams") as opposed to the two-stream (Pulliainen et al., 1999) or six-stream (Wiesmann and Mätzler, 1999) methods whose formulations are based on a fixed and small number of directions. The drawback of the DISORT method is usually the computational cost. However, in the case of passive remote sensing, isotropic media, and when the phase function has a simple analytical form, the azimuthal dependence in Eq. (1) can be analytically integrated. This simplifies the equation and reduces the numerical complexity and computation cost with respect to other cases like active remote sensing (Stamnes et al., 1988; Picard et al., 2004) or anisotropic media. For snow passive microwave modeling, the assumption of an isotropic medium is reasonable and the DMRT theory with small scatterers predicts that the phase function is the Rayleigh phase function (Jin, 1994, Eqs. 2-26 to 2-28), which only involves the cosines of the azimuth angle.

In the single layer formulation of DISORT, the integration over the zenith angle $\theta^{\prime}$ uses a Gaussian quadrature (Jin, 1994, p. 96), i.e., it is replaced by a discrete sum of integrand evaluation at optimal angles. The number of angles $n$ is defined by the user. This approach cannot be seamlessly transposed for multiple layers. The variations of refractive index - mainly driven by the snow density profile - cause a change of the direction of the streams between the layers (Fig. 2). A possible approach (Liang et al., 2008) uses the same Gaussian quadrature in every layer (as in the singlelayer case), which ensures an optimal integration at the expense of complex boundary conditions since cubic spline interpolations are needed to "reconnect" the streams. We use a simpler approach in DMRT-ML similar to that proposed in Jin (1994, p. 151). The angles are determined by Gaussian quadrature only in the most refractive layer. In the other layers, the angles are determined from Snell's refraction law, which ensures the continuity of the streams between the layers. The boundary conditions are simpler at the expense of a sub-optimal integration (except in the most refractive layer). This issue is easily compensated by increasing the number of streams $n$.

Another consequence of using the refraction law to determine the stream angles is that the number of streams $\left(n_{k}\right.$, $k=1 \cdots L$, where $L$ is the number of layers) varies from one layer to another. This is caused by the total reflection at large zenith angles when a stream propagates from a higher to a lower refractive index layer. Such streams in the high refractive index layer have no companion in the low index one. This also applies to the $n_{0}$ streams emerging from the snow into the air. Since snow density is much higher than air density, $n_{0}$ is usually much lower than $n$. The only consequence for the user is that $n$ is not the number of emerging streams as it would be in the six-stream or two-stream methods. In practice, it is recommended to adjust $n$ to get the wanted number of emerging streams $n_{0}$ or alternatively to increase $n$ until the residual variations of brightness temperature are less than the wanted accuracy. Figure 2 illustrates a 4-layer snowpack (with snow density values, from top to bottom, of 50, 400, $200,320 \mathrm{~kg} \mathrm{~m}^{-3}$ ). Only upwelling streams are represented. 


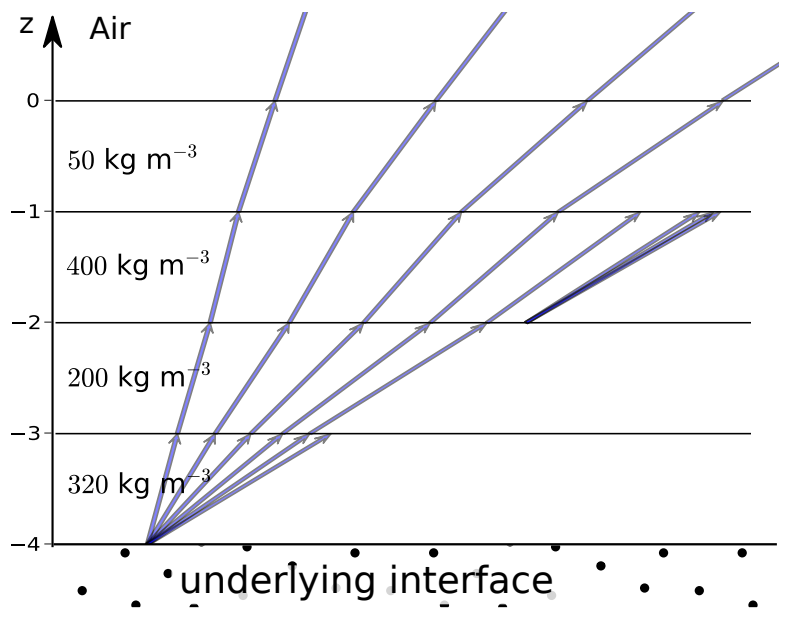

Fig. 2. Upwelling streams for a 4-layer snowpack with varying density. The stream directions are calculated with Snell's refraction law using the real part of the effective propagation constant $E_{\text {eff }}$ (Eq. 6) that mostly depends on snow density. The number of streams is 8 in the most dense layer and decreases as the density decreases. Only 4 streams emerge in the air above the snowpack. For clarity, downwelling streams are not represented.

In this particular example with extreme variations of density, the number of streams in the air is only 4 whereas it is 8 in the most refractive layer. The total reflection phenomenon is also called internal reflection in Wiesmann and Mätzler (1999) and causes energy trapping when a layer is surrounded by less dense layers.

Once the integration is replaced by the discrete sum, the differential Eq. (1) in $z$ is written for every angle $\theta_{j}(j=$ $\pm 1 \cdots \pm n_{k}, j>0$ and $j<0$ for the upward and downward directions, respectively) in each layer.

$$
\begin{aligned}
\mu_{j} & \frac{\mathrm{d}}{\mathrm{d} z} \boldsymbol{T}_{\mathrm{B}}\left(\mu_{j}, z\right)=-\kappa_{\mathrm{e}} \boldsymbol{T}_{\mathrm{B}}\left(\mu_{j}, z\right) \\
& +\sum_{j^{\prime}}^{n_{k}} w_{j^{\prime}} \mathbf{P}\left(\mu_{j}, \mu_{j^{\prime}}\right) \boldsymbol{T}_{\mathrm{B}}\left(\mu_{j}^{\prime}, z\right)+\kappa_{\mathrm{a}} T \boldsymbol{I}
\end{aligned}
$$

where $\mu_{j}=\cos \left(\theta_{j}\right), \mathbf{P}$ is found in Jin (1994, Eq. 2-28) and $\boldsymbol{I}$ is the unit column vector. In the following, we only present the most relevant equations, intermediate calculations are given in Jin (1994, Chaps. 4 and 5).

By introducing the asymmetric and symmetric brightness temperatures $\left(\boldsymbol{T}_{\mathrm{Bs}}\left(\mu_{j}\right)=\boldsymbol{T}_{\mathrm{B}}\left(\mu_{j}\right)+\boldsymbol{T}_{\mathrm{B}}\left(-\mu_{j}\right)\right.$ and $\boldsymbol{T}_{\mathrm{Ba}}\left(\mu_{j}\right)=\boldsymbol{T}_{\mathrm{B}}\left(\mu_{j}\right)-\boldsymbol{T}_{\mathrm{B}}\left(-\mu_{j}\right)$ respectively), Eq. (9) becomes

$$
\begin{aligned}
& \mu_{j} \frac{\mathrm{d}}{\mathrm{d} z} \boldsymbol{T}_{\mathrm{Ba}}\left(\mu_{j}, z\right)=-\kappa_{\mathrm{e}} \boldsymbol{T}_{\mathrm{Bs}}\left(\mu_{j}, z\right), \\
& \mu_{j} \frac{\mathrm{d}}{\mathrm{d} z} \boldsymbol{T}_{\mathrm{Bs}}\left(\mu_{j}, z\right)=\sum_{j^{\prime}}^{n_{k}} \mathbf{A}_{j j^{\prime}} \boldsymbol{T}_{\mathrm{Ba}}\left(\mu_{j}^{\prime}, z\right)+2 \kappa_{\mathrm{a}} T, \\
& \mathbf{A}_{j j^{\prime}}=-\kappa_{\mathrm{e}} \mathbf{I} \delta_{j j^{\prime}}+\frac{3}{4} \kappa_{\mathrm{s}} w_{j^{\prime}}\left[\begin{array}{rr}
2\left(1-\mu_{j}^{2}\right)\left(1-\mu_{j^{\prime}}^{2}\right)+\mu_{j}^{2} \mu_{j^{\prime}}^{2} \\
\mu_{j^{\prime}}^{2} & \mu_{j}^{2} \\
1
\end{array}\right]
\end{aligned}
$$

where $\mathbf{I}$ is the $2 \times 2$ identity matrix and $\delta_{j j^{\prime}}$ is 1 when $j=j^{\prime}$, otherwise 0. Note that the $\mathbf{W}$ matrix in Jin (1994, Eq. 4-42b) is trivial in our case $\mathbf{W}=-\kappa_{e}$ owing to the symmetry of the Rayleigh phase function. Differentiating Eq. (10a) and combining it with Eq. (10b) leads to a second-order ordinary system of differential equations. The solutions are of the form

$$
\begin{aligned}
& \boldsymbol{T}_{\mathrm{Ba}}\left(\mu_{j}, z\right) \\
& =\sum_{m}^{2 n_{i}}\left[x_{m} \exp \left(\alpha_{m} z\right)+y_{m} \exp \left(-\alpha_{m}(z+d)\right)\right] \boldsymbol{T}_{\mathrm{Ba}, m}^{0}\left(\mu_{j}\right)+2 T \boldsymbol{I},
\end{aligned}
$$

where $d$ is the layer depth, $\alpha_{m}=+\sqrt{\Lambda_{m}},\left(m=1 \ldots 2 n_{i}\right)$ and $\Lambda_{m}$ are the eigenvalues and $\boldsymbol{T}_{\mathrm{B} a, m}^{0}\left(\mu_{j}\right)$ the eigenvectors of the $2 n_{i} \times 2 n_{i}$ matrix whose elements are $\frac{\kappa_{e}}{\mu_{j}^{2}} \mathbf{A}_{j j^{\prime}}^{p p^{\prime}}$ $\left(j=1 \ldots N, p=v, h\right.$ and $\left.j^{\prime}=1 \ldots N, p^{\prime}=v, h\right) . x_{m}$ and $y_{m}$ are $2 \times 2 n_{i}$ unknown constants to be determined with the boundary conditions. In DMRT-ML, the eigenvalue problem is solved using LAPACK routines (Anderson et al., 1999).

Combining Eqs. (10b) and (11), the solutions for the symmetric brightness temperature are

$$
\begin{aligned}
\boldsymbol{T}_{\mathrm{Bs}}\left(\mu_{j}, z\right) & =\sum_{m}^{2 n_{i}}\left[x_{m} \exp \left(\alpha_{m} z\right)\right. \\
& \left.-y_{m} \exp \left(-\alpha_{m}(z+d)\right)\right] \boldsymbol{T}_{\mathrm{Bs}, m}^{0}\left(\mu_{j}\right), \\
\boldsymbol{T}_{\mathrm{Bs}, m}^{0}\left(\mu_{j}\right) & =\sum_{j^{\prime}}^{n_{k}} \frac{1}{\mu_{j} \alpha_{m}} \mathbf{A}_{j j^{\prime}} \boldsymbol{T}_{\mathrm{Ba}, m}^{0}\left(\mu_{j^{\prime}}\right) \\
& =\kappa_{\mathrm{e}} \mu_{j} \alpha_{m} \boldsymbol{T}_{\mathrm{Ba}, m}^{0}\left(\mu_{j}\right) .
\end{aligned}
$$

Note that $\boldsymbol{T}_{\mathrm{Ba}, m}^{0}\left(\mu_{j}\right)$ and $\boldsymbol{T}_{\mathrm{Bs}, m}^{0}\left(\mu_{j}\right)$ are written $E$ and $Q$, respectively, in Jin (1994, p. 102).

The boundary conditions express the conservation of energy at every interface. For each layer $k$ of depth $d_{k}$, every direction $j$ and polarization, there are two boundary conditions (Jin, 1994, Eqs. 5-10a and 5-10c). The boundary condition at the top interface is

$$
\begin{array}{r}
\sum_{m}^{n_{k}}\left[\left(1-\mathbf{r}_{j, k}^{\mathrm{top}}\right) \boldsymbol{T}_{\mathrm{Ba}, m, k}^{0}\left(\mu_{j, k}\right)\right. \\
\left.-\left(1+\mathbf{r}_{j, k}^{\mathrm{top}}\right) \boldsymbol{T}_{\mathrm{Bs}, m, k}^{0}\left(\mu_{j, k}\right)\right] x_{m, k} \\
+\left[\left(1-\mathbf{r}_{j, k}^{\mathrm{top}}\right) \boldsymbol{T}_{\mathrm{Ba}, m, k}^{0}\left(\mu_{j, k}\right)+\right. \\
\left.\left(1+\mathbf{r}_{j, k}^{\mathrm{top}}\right) \boldsymbol{T}_{\mathrm{Bs}, m, k}^{0}\left(\mu_{j, k}\right)\right] \exp \left(-\alpha_{m, k} d_{k}\right) y_{m, k} \\
-\sum_{m}^{n_{k-1}}\left(1-\mathbf{r}_{j, k}^{\mathrm{top}}\right)\left[\boldsymbol{T}_{\mathrm{Ba}, m, k-1}^{0}\left(\mu_{j, k}\right)\right. \\
\left.-\boldsymbol{T}_{\mathrm{Bs}, m, k-1}^{0}\left(\mu_{j, k}\right)\right] \exp \left(-\alpha_{m, k-1} d_{k-1}\right) x_{m, k-1} \\
-\left(1-\mathbf{r}_{j, k}^{\mathrm{top}}\right)\left[\boldsymbol{T}_{\mathrm{Ba}, m, k-1}^{0}\left(\mu_{j, k}\right)\right. \\
\left.+\boldsymbol{T}_{\mathrm{Bs}, m, k-1}^{0}\left(\mu_{j, k}\right)\right] y_{m, k-1} \\
=\left(1-\mathbf{r}_{j, k}^{\mathrm{top}}\right)\left(T_{k-1}-T_{k}\right),
\end{array}
$$


and the one at the bottom interface is

$$
\begin{array}{r}
\sum_{m}^{n_{k+1}}\left(1-\mathbf{r}_{j, k}^{\mathrm{bottom}}\right)\left[\boldsymbol{T}_{\mathrm{Ba}, m, k+1}^{0}\left(\mu_{j, k}\right)\right. \\
\left.+\boldsymbol{T}_{\mathrm{Bs}, m, k+1}^{0}\left(\mu_{j, k}\right)\right] x_{m, k+1} \\
+\left(1-\mathbf{r}_{j, k}^{\mathrm{bottom}}\right)\left[\boldsymbol{T}_{\mathrm{Ba}, m, k+1}^{0}\left(\mu_{j, k}\right)\right. \\
\left.-\boldsymbol{T}_{\mathrm{Bs}, m, k+1}^{0}\left(\mu_{j, k}\right)\right] \exp \left(-\alpha_{m, k+1} d_{k+1}\right) y_{m, k+1} \\
-\sum_{m}^{n_{k}}\left[\left(1-\mathbf{r}_{j, k}^{\mathrm{bottom}}\right) \boldsymbol{T}_{\mathrm{Ba}, m, k}^{0}\left(\mu_{j, k}\right)\right. \\
\left.+\left(1+\mathbf{r}_{j, k}^{\mathrm{bottom}}\right) \boldsymbol{T}_{\mathrm{Bs}, m, k}^{0}\left(\mu_{j, k}\right)\right] \exp \left(-\alpha_{m, k} d_{k}\right) x_{m, k} \\
-\left[\left(1-\mathbf{r}_{j, k}^{\mathrm{bottom}}\right) \boldsymbol{T}_{\mathrm{Ba}, m, k}^{0}\left(\mu_{j, k}\right)\right. \\
\left.-\left(1+\mathbf{r}_{j, k}^{\mathrm{bottom}}\right) \boldsymbol{T}_{\mathrm{Bs}, m, k}^{0}\left(\mu_{j, k}\right)\right] y_{m, k} \\
=\left(1-\mathbf{r}_{j, k}^{\mathrm{bottom}}\right)\left(T_{k}-T_{k+1}\right),
\end{array}
$$

where $\mathbf{r}_{j, k}^{\text {top }}$ and $\mathbf{r}_{j, k}^{\text {bottom }}$ are the reflection coefficients for streams going from the layer $k$ to the upper $k-1$ and lower $k+1$ layers respectively. At the top of the snowpack, Eq.(13) is formally valid if $x_{0}$ and $y_{0}$ are set to zero and $T_{0}$ is $T_{\mathrm{B}}^{\text {atmos }}$. At the bottom of the snowpack, Eq. (14) is valid if $x_{L+1}$ and $y_{L+1}$ are set to 0 , the reflection coefficients $\mathbf{r}_{j L}^{\text {bottom }}$ are calculated with the substratum model (Table 1) and $T_{L+1}$ is the substratum temperature.

The set of boundary conditions forms a linear system of $N$ equations $\left(N=4 \sum_{k} n_{k}\right)$. Since the unknowns $x$ and $y$ in layer $k$ are only linked to unknowns in layer $k-1$ and $k+1$, the system can be arranged in block-diagonal structure and can be solved using the efficient banded matrix solver in LAPACK. The brightness temperature emerging from the snowpack is then calculated with

$$
\begin{array}{r}
\boldsymbol{T}_{\mathrm{B}}\left(\mu_{j, 0}, z=0\right)=\left(1-\mathbf{r}_{j, 0}^{\mathrm{bottom}}\right)\left(T_{1}+\sum_{m}^{n_{1}}\right. \\
{\left[\boldsymbol{T}_{\mathrm{Ba}, m, 1}^{0}\left(\mu_{j, 0}\right)+\boldsymbol{T}_{\mathrm{Bs}, m, 1}^{0}\left(\mu_{j, 0}\right)\right] x_{m, 1}} \\
+\left[\boldsymbol{T}_{\mathrm{Ba}, m, 1}^{0}\left(\mu_{j, 1}\right)-\boldsymbol{T}_{\mathrm{Bs}, m, 1}^{0}\left(\mu_{j, 1}\right)\right] \\
\left.\exp \left(-\alpha_{m, 1} d_{1}\right) y_{m, 1}\right) .
\end{array}
$$

The value from this last equation is returned to the user as the simulated top-of-snowpack brightness temperature.

\subsection{Post-computation: emissivity and reflectivity}

The emissivity, i.e., the coefficient measuring the departure from a black body, is often used to present modeling results in passive microwave studies instead of brightness temperature (whose value is related to the snow physical temperature). Only when the medium is strictly isothermal, that is, when the physical temperature of snow and the underlying medium is uniform and equal to $T$, it is possible to compute the emissivity using a single simulation and the following equation:

$\boldsymbol{e}=\frac{\boldsymbol{T}_{\mathrm{B}}}{T}$.

For a nonconstant temperature profile, which is the rule for any natural snowpack, the definition of emissivity is not trivial. To mimic the in-equilibrium case and the Kirchhoff law, the emissivity can be defined as one minus the reflectivity of the medium (Peake, 1959). The calculation requires two simulations with slightly different atmospheric brightness temperatures $\left(T_{\mathrm{B}}^{\mathrm{atmos}}\right.$ and $\left.T_{\mathrm{B}}^{\mathrm{atmos}}+\Delta T_{\mathrm{B}}^{\mathrm{atmos}}\right)$. Assuming $\boldsymbol{T}_{\mathrm{B}}$ and $\boldsymbol{T}_{\mathrm{B}}^{\prime}$ are the results of these simulations, the reflectivity and emissivity are given by

$\boldsymbol{e}=1-\boldsymbol{r}=1-\frac{\boldsymbol{T}_{\mathrm{B}}^{\prime}-\boldsymbol{T}_{\mathrm{B}}}{\Delta T_{\mathrm{B}}^{\mathrm{atmos}}}$.

In practice, using $\boldsymbol{T}_{\mathrm{B}}^{\mathrm{atmos}}=0$ and $\Delta T_{\mathrm{B}}^{\mathrm{atmos}}=1 \mathrm{~K}$ is recommended.

\section{Results}

This section presents the sensitivity of DMRT-ML to the most important snow properties required as inputs. It also discusses the limitations of the model and provides the range of validity of the input variables whenever possible.

\subsection{Sensitivity to ice dielectric constant}

Figure 3 a shows brightness temperatures at $18 \mathrm{GHz}$ as a function of the zenith angle calculated with DMRT-ML and calculated by Kong et al. (1979) together with observations reported by Tsang and Kong (2001, Fig. 7.7.2). The medium is considered semi-infinite, the grain size was chosen to be $1.75 \mathrm{~mm}$ and the ice dielectric constant $\varepsilon_{\text {ice }}=3.2+i 0.016$. The result of the DMRT-ML simulation with prescribed dielectric constant (solid line) closely matches the original calculation (dotted line) by Tsang and Kong (2001) up to incidence angles of $65^{\circ}$. This provides a technical validation of our implementation in the single layer case. However, the imaginary part of the ice dielectric constant used by Kong et al. (1979) was an order of magnitude higher than the one obtained with the more recent Eq. (2) from Mätzler and Wegmüller (1987). With the latter parameterization, the brightness temperatures simulated using the same grain size of $1.75 \mathrm{~mm}$ are much lower (dash line in Fig. 3b), leading to a strong disagreement with the observations. Modeling results and observations can be re-conciliated by using a smaller radius of $0.83 \mathrm{~mm}$ (solid line in Fig. 3b). This new simulation yields results very close to those of the original simulation and observations.

Even if Eq. (2) is likely closer to reality than earlier formulas, the parameters of ice dielectric constants are still not 

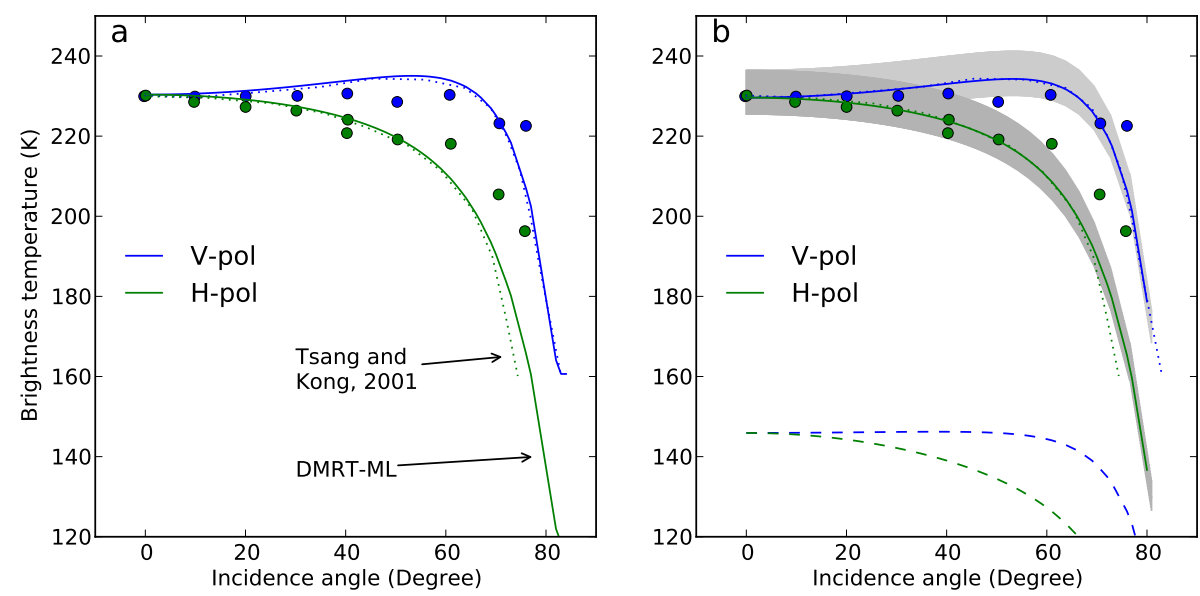

Fig. 3. Comparison of DMRT-ML simulations (solid line) at $18 \mathrm{GHz}$ with previous calculations (Tsang and Kong, 2001) (dotted curve) and with experimental observations (filled dots). The medium is semi-infinite, with a density of $350 \mathrm{~kg} \mathrm{~m}^{-3}$, a temperature of $272 \mathrm{~K}$, a grain radius of $1.75 \mathrm{~mm}$ and an ice dielectric constant of $3.2+\jmath 0.016$. Simulations with a more realistic dielectric constant (Mätzler and Wegmüller, 1987) are shown on the right panel with the original radius of $1.75 \mathrm{~mm}$ (dashed curve) and refitted radius of $0.83 \mathrm{~mm}$ (solid line). The gray bars represent variations of the dielectric constant imaginary part of $\pm 20 \%$ around in the later case.

well constrained by measurements especially at low frequencies (Jiang and $\mathrm{Wu}, 2004)$ and the uncertainty is unknown. The consequences on the predicted brightness temperature can be significant as illustrated in Fig. 3b (gray area) obtained by assuming an error of $\pm 20 \%$ of the dielectric loss (imaginary part of the dielectric constant). At a $53^{\circ}$ incidence angle for instance, the error is 11 and $12 \mathrm{~K}$ at vertical and horizontal polarizations respectively. Such an error is significant and must be taken into account in the interpretation of simulations by thermal microwave emission models.

\subsection{Sensitivity to the grain size}

The significant sensitivity of microwave thermal radiation to snow grain size is widely recognized (Zwally, 1977). It stems from the fact that snow grains are usually smaller than the wavelength in the microwave range and their scattering coefficient (Eq. 8c) increases as the cubic power of the sphere radius $a$. Figure 4 presents the variation of the scattering efficiency $\left(Q_{\mathrm{s}}=4 a \kappa_{\mathrm{s}} / 3 f\right)$ as a function of sphere radius. The calculations with DMRT-ML (for various densities) are plotted as solid lines.

In contrast, the absorption coefficient increases linearly with the size. It results that the emissivity and the brightness temperature of a semi-infinite medium strongly decrease with size (Fig. 5). The difference between vertical and horizontal polarizations also slightly decreases as scattering increases.

An important consequence of this cubic dependence is that a collection of spheres with different sizes is not equivalent to a collection of identical spheres with the averaged size. The contribution of the largest spheres of the collection to scattering is comparably greater than the contribution of the small- est. However, Jin (1994) shows that, under the assumption of small grains, any collection can be represented by equalradius spheres with an equivalent grain size $a_{c}$. This size is always larger than the average of the distribution but depends on the distribution shape as well as snow density (Jin, 1994, Eq. 3-42 and Figs. 3-11, 3-12). In DMRT-ML, we implemented the calculation for a Rayleigh distribution of size and reached the same conclusion as Jin (1994). Unfortunately, the results are highly dependent on the choice of the distribution and especially on the slope of the upper tail of the distribution. For instance, a log-normal distribution - relevant for snow (Flanner and Zender, 2006) - features many very large grains for a reasonable mean grain size, which leads to the divergence of the DMRT-ML calculation and breaks the assumptions of small scatterers. In practice, the choice of the distribution is difficult and is related to the more general issue of the representation of snow by a collection of spherical grains.

Figure 4 also illustrates the empirical correction for large particles proposed in DMRT-ML (Sect. 2.3). The corrected scattering efficiency (circles) is limited to a maximum value of 2 , which corresponds to the theoretical asymptotic value for very large particles (Grody, 2008). This correction eliminates the unrealistic divergence of the scattering efficiency for large grain sizes (solid line). Nevertheless, the quality of this correction is difficult to evaluate for dense media. For a sparse medium however, the scatterers are independent and the scattering efficiency is obtained by Mie's calculation (Warren, 1982). Figure 4 (dashed blue curve) shows that the Mie scattering efficiency at $89 \mathrm{GHz}$ diverges from DMRTML with density tending to $0 \mathrm{~kg} \mathrm{~m}^{-3}$ (i.e., independent scatterers) for grain radii larger than $0.75 \mathrm{~mm}$ (i.e., $a \approx \lambda / 5$ and $Q_{\mathrm{s}}$ of 2-3). This is in agreement with our correction. 


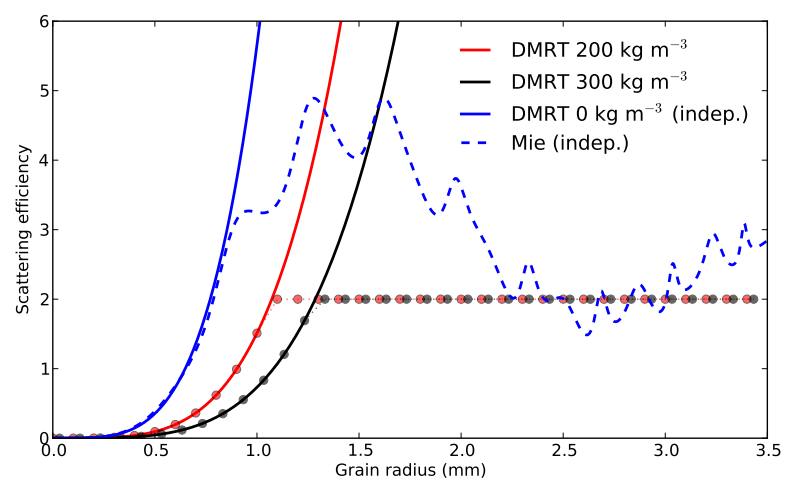

Fig. 4. Scattering efficiency $\left(Q_{\mathrm{s}}=4 a \kappa_{\mathrm{s}} / 3 f\right)$ at $89 \mathrm{GHz}$ as a function of the grain size for different approximations and densities: Independent scattering approximation (applies for very small density only) using DMRT-ML with a density approaching 0 (solid blue line), or full Mie calculation (dashed blue line); DMRT-ML QCA-CP nonsticky with a density of $200 \mathrm{~kg} \mathrm{~m}^{-3}$ (solid red line) and $300 \mathrm{~kg} \mathrm{~m}^{-3}$ (solid black line). Dotted curves show calculations based on the DMRT-ML theory with a limitation of the scattering efficiency at $Q_{\mathrm{s}}=2$, for two different densities.

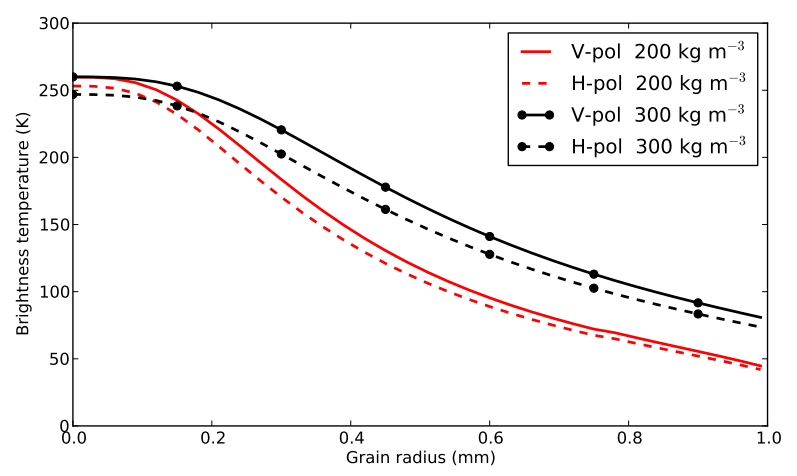

Fig. 5. Brightness temperature at $89 \mathrm{GHz}, 53^{\circ}$ incidence angle, horizontal and vertical polarizations (dashed and solid line, respectively) as a function of the grain size with a density of 200 (red) and $300 \mathrm{~kg} \mathrm{~m}^{-3}$ (black).

However, Mie efficiency reaches a maximum value of nearly 5 and remains largely above 2 in the range of realistic grain sizes. This is not reproduced by our correction. In fact, the convergence towards 2 is only observed at much larger grain sizes. This result suggests that the empirical correction would be more accurate if the efficiency limit were increased up to a value around 4. However, this result was derived in particular conditions (sparse medium, $89 \mathrm{GHz}$ ) and its generality is unknown. We therefore recommend to use the correction with caution and only when a very limited number of layers in the snowpack have large grains. It is worth noting that the condition $Q_{\mathrm{s}}<2$ is valid for most types of snow at frequencies lower or equal to $89 \mathrm{GHz}$ as shown in Fig. 6. Harlow and Essery (2012, Fig. 11) show using Mie-QCA with sticky spheres $(\tau=0.2)$ that this assumption is valid up to about $60 \mathrm{GHz}$ for $1 \mathrm{~mm}$-radius particles and to $200 \mathrm{GHz}$ for particles of $0.3 \mathrm{~mm}$ radius and smaller. This confirms that the

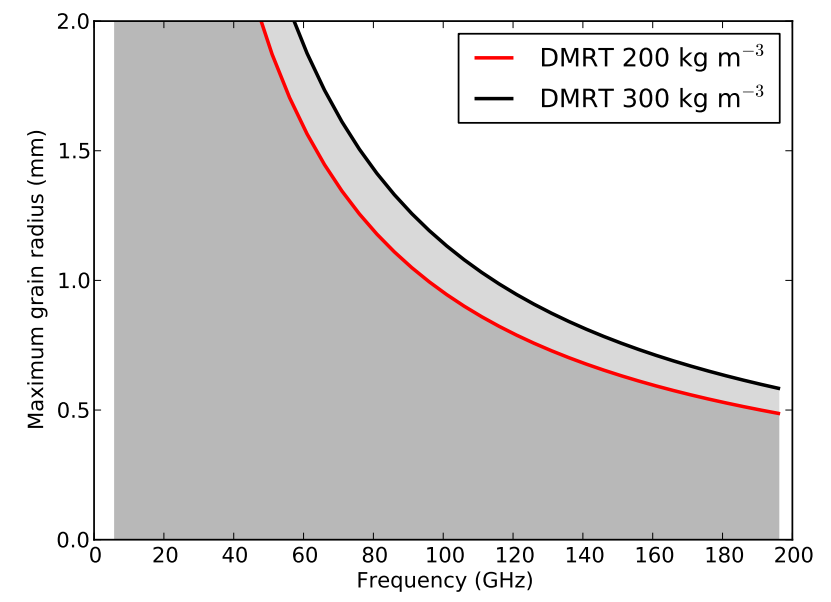

Fig. 6. Range of grain sizes for which the DMRT-ML QCA-CP nonsticky is reasonably valid as a function of the frequency for snow at $260 \mathrm{~K}$ and 200 (red) or $300 \mathrm{~kg} \mathrm{~m}^{-3}$ (black). The upper limit is the grain size for which the scattering efficiency reaches a value of 2 .

general criteria $a \lesssim \lambda / 5$ is adequate to evaluate the validity of QCA-CP for small scatterers.

However, even if the condition $Q_{\mathrm{s}}<2$ is true, we note that the brightness temperatures become unrealistically low before $Q_{\mathrm{s}}$ reaches a value of 2 (which occurs for $a>1 \mathrm{~mm}$ in Fig. 5). For example, the lowest brightness temperature ever observed at $89 \mathrm{GHz}$ in Antarctica by AMSR-E is $117 \mathrm{~K}$.

\subsection{Influence of snow density}

Snow density is involved in many components of the model. It drives (i) the proximity of the grains, thus the scattering coefficient of the medium in relation with the grain size (Eqs. 5, 6); (ii) the mass of ice, thus the absorption coefficient; (iii) the real part of the refractive index, which determines the stream angles, and the transmission and reflection coefficients of every interface (West et al., 1996).

The first two effects are illustrated in Fig. 7 at $37 \mathrm{GHz}$ and for a grain size of $0.3 \mathrm{~mm}$. The absorption and scattering coefficients are calculated assuming a medium of "ice spheres in air" for density less than half of the pure ice density (i.e., fractional volume of $50 \%$ ) and "air spheres in ice" otherwise. The discontinuity observed in Fig. 7 comes from the fact that both representations are not strictly equivalent even at a fractional volume of $50 \%$.

The absorption coefficient increases almost linearly with the density because the ice is the only absorber. In contrast, the case of the scattering coefficient is more complex. For very low density, the medium is sparse and the scattering coefficient calculated with the DMRT-ML theory increases closely to the one calculated with the independent scatterers assumption (Fig. 7, dashed line). However, for densities larger than $100 \mathrm{~kg} \mathrm{~m}^{-3}$, the latter becomes invalid, because the scatterers are too close to be considered independent - 
they are in fact in the shadow of each other, which weakens their scattering efficiency (Liang et al., 2006). This effect is nicely captured by the DMRT-ML theory (Tsang et al., 2000b, Fig. 10.4.5), which predicts that the rate of variation of the scattering coefficient decreases with density. The coefficient reaches a maximum at a density around $150 \mathrm{~kg} \mathrm{~m}^{-3}$ and decreases for higher densities. Although this general behavior is expected, the accuracy of the DMRT-ML theory is not well known at such high densities. Recent work using exact numerical calculation has shown that the theory DMRT-ML QCA starts to deviate from fractional volume around $30 \%$ (Liang et al., 2006), i.e., a density of $275 \mathrm{~kg}$ $\mathrm{m}^{-3}$ (validity range represented by circles in Fig. 7). However, the generality of this result for smaller grains, moderate stickiness or under the QCA-CP assumption is unexplored. If we assume that the value of $30 \%$ is correct and applies also for "air spheres in ice", the theory would be valid in the range $640-917 \mathrm{~kg} \mathrm{~m}^{-3}$ (represented by squares in Fig. 7). Unfortunately, snow density falls in the intermediate range $\left(275-640 \mathrm{~kg} \mathrm{~m}^{-3}\right)$ in many conditions. To deal with this issue in practice, a pragmatic option is to consider the deviation above $30 \%$ fractional volume is moderate with respect to other errors (such as grain size measurements) and to apply DMRT-ML QCA-CP using the most adequate medium representation as a function of the density as in Fig. 7. A second option is to interpolate the scattering and absorption coefficients using polynomials fitted with anchor points taken in both domains where the theory is valid. This option called "bridging" (Dierking et al., 2012) is appealing because it yields continuous relationships as a function of the density. However, the sensitivity to the choice of the polynomial order and the anchor points has to be evaluated. Therefore, the "bridging" option is not implemented in DMRT-ML yet.

\subsection{Influence of the stickiness}

Figure 8 shows the scattering coefficient at $37 \mathrm{GHz}$ as a function of density for different values of the stickiness parameter $\tau$ and grain radius $a$ for a given temperature. It shows that the scattering coefficient increases as the stickiness parameter $\tau$ decreases (from blue to black to green curves). The lower values of stickiness correspond indeed to stronger attractions between spheres and a more pronounced clustering effect. Clusters are "seen" by the microwaves as larger objects than the particles they are made of. It means that they scatter more than if the particles were randomly positioned (i.e. nonsticky case). However, the stickiness parameter is not just a scaling factor of the grain size. In fact, a cluster of small particles is not equivalent to a large particle as illustrated in Fig. 8: the sensitivity to the density is different between a cluster (green curve) and a large particle (red curve). The stickiness tends to shift the maximum of the scattering coefficient toward a larger density.

In practice, choosing a realistic value of stickiness to represent natural snow is difficult. There is currently no means

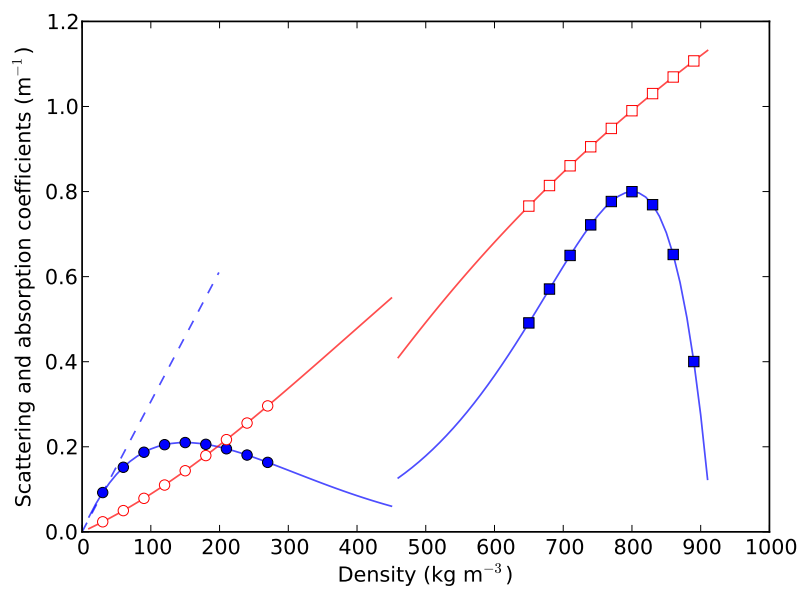

Fig. 7. Scattering (blue and plain symbols) and absorption (red and hollow symbols) coefficients at $37 \mathrm{GHz}$ as a function of the density. The temperature is $260 \mathrm{~K}$ and the grain radius is $0.3 \mathrm{~mm}$. The model "ice spheres in air" is used for densities less than half the pure ice density $\left(458.5 \mathrm{~kg} \mathrm{~m}^{-3}\right)$. For higher densities, the model "air spheres in ice" is used. Circles and squares show the domain of validity of the DMRT-ML theory for each model. The scattering coefficient under the assumption of independent "ice spheres in air" is shown as a blue dash curve.

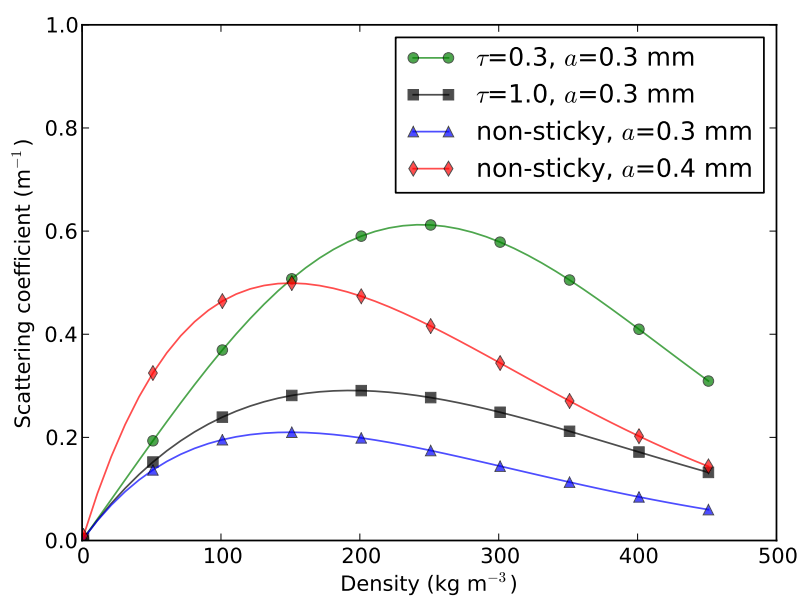

Fig. 8. Scattering coefficient at $37 \mathrm{GHz}$ as a function of the density for different stickiness parameters $\tau$ and grain radius $a$. The temperature is $260 \mathrm{~K}$.

to estimate this value either from field measurements, 3-D images of snow micro-structure or snow evolution model outputs. As for choosing a grain size distribution, the core of the problem is the representation of snow by spheres. Tsang et al. (2008) suggest to use $\tau=0.1$ because it yields a frequency-dependence in better agreement with measurements. With such a low value, the clustering effect is in fact very strong and the size of the cluster approaches the wavelength (long-range effect), which explains the change of the frequency-dependence. Mätzler (1998) suggests to use a higher value, $\tau=0.2$, based on a comparison between the 
density dependence in DMRT-ML with various degrees of stickiness and in the improved Born approximation (Mätzler, 1998).

The implementation of the stickiness in DMRT-ML is limited to the "short range" version, i.e., both the grains and the cluster must be small with respect to the wavelength. In practice, $\tau$ should be larger than its theoretical minimum value of $(2-\sqrt{2}) / 6=0.098$ (Tsang et al., 2000b, p. 427). After Tsang et al. (2000b, Fig. 8.4.3) and our own calculation (not shown), the "short range" calculation is valid for $\tau$ values larger than 0.25 and grain sizes, which respects the small scatterer assumption. For lower $\tau$, the validity depends on the grain size.

\subsection{Influence of the liquid water content}

Figure 9 shows the relationship between the brightness temperature at $19 \mathrm{GHz}$ and horizontal polarization as a function of the total column liquid water content. The snowpack is considered homogeneous, except that the liquid water is concentrated in the top $10 \mathrm{~cm}$. This calculation confirms the strong influence of the liquid water on brightness temperature, which is exploited to detect snowmelt events from passive microwave observations (e.g. Picard and Fily, 2006). It also shows that brightness temperature reaches a nearly constant value from about $0.5 \mathrm{~kg} \mathrm{~m}^{-2}$ of liquid water. This feature explains why the retrieval of the amount of liquid water from passive microwave observation is probably impossible. The threshold value, $0.5 \mathrm{~kg} \mathrm{~m}^{-2}$, is close to value obtained with the MEMLS model (Tedesco et al., 2007) and that obtained indirectly by comparing observations and outputs of the RACMO regional snow and atmosphere model (Kuipers Munneke et al., 2012).

\subsection{Layered snowpack}

It is well known that the natural snowpack is composed of relatively homogeneous layers formed by episodes of accumulation and subject to metamorphisms. Hence, the density and grain size (and stickiness) can be different in every layer. Since the electromagnetic properties (i.e., the scattering and absorption coefficients and the effective constant of propagation) have a nonlinear dependence to the snow properties, the single-layer "average" snowpack is not electromagnetically equivalent to the multi-layer snowpack. In addition, the temperature is rarely uniform within the snowpack, especially close to the surface where the strong temperature gradients are caused by the daily variations of solar energy (Brandt and Warren, 1993).

Several effects can result from the layered nature of the snowpack. In general, accounting for the multi-layered nature is particularly important for

- the difference between the horizontal and vertical polarizations. The difference is particularly sensitive to reflection at the air-snow interface, which is driven by the

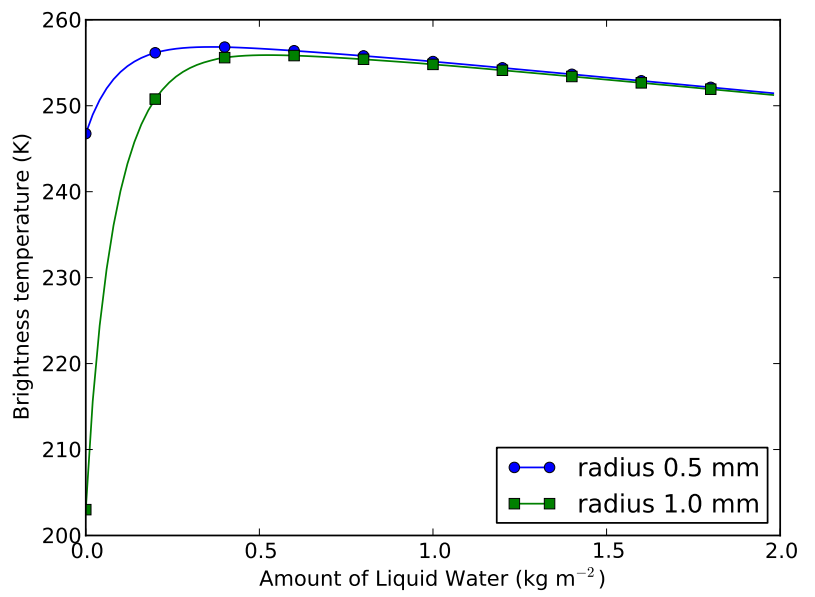

Fig. 9. Brightness temperature at $19 \mathrm{GHz}$ and horizontal polarization as a function of the total amount of liquid water in the snowpack. The temperature is $273 \mathrm{~K}$, density is $300 \mathrm{~kg} \mathrm{~m}^{-3}$ and there exist two grain size values $(0.5$ and $1 \mathrm{~mm})$. The liquid water is concentrated in the top first $10 \mathrm{~cm}$ of the snowpack.

density in the upper layer and by the reflection at the internal interface, which depend on the contrast of density between successive layers. There are several experimental and theoretical evidences of this effect (Mätzler et al., 1984; Liang et al., 2008; Champollion et al., 2013). Smoothing the profile of density in simulation results directly in a decrease of the difference between the polarizations.

- the spectrum of emissivity. It is particularly sensitive to the scattering and absorption profiles because the penetration depth highly depends on the frequency. For instance, Brucker et al. (2010) showed that the spectra observed in Antarctica cannot be explained with a homogeneous snowpack, and that the increase of grain size with depth is a necessary condition to explain the observed spectra. In some extreme cases called "anomalous spectra" by Rosenfeld and Grody (2000), the observed emissivity increases with frequency although the emissivity of a homogeneous snowpack decreases with frequency due to a stronger increase of scattering relative to that of the absorption. To illustrate the effect of the resolution of the snow parameter profiles, the calculation presented in Brucker et al. (2011a) using the original $2.5 \mathrm{~cm}$ high-resolution profiles of grain size and density measured at Dome $\mathrm{C}$ in Antarctica are presented in Fig. 10 along with calculations using lowerresolution profiles. The simulations are performed at 19 and $37 \mathrm{GHz}$ with a homogeneous temperature of $219 \mathrm{~K}$ (the annual mean at Dome C). Only the resolution in the upper $3 \mathrm{~m}$ for which measurements were available is varied. Figure 10 shows that coarser-resolution results in higher brightness temperatures (up to about $8 \%$ ), except for the lowest resolution (3 m-thick layer, 
corresponding to the homogeneous snowpack). This complex dependence might be explained by the fact that the modeled brightness temperature is more sensitive to the resolution of the density profile than that of the grain size (simulations not shown) and that the density dependence of the electromagnetic properties is not monotonic as shown in Fig. 7. These results are specific to Dome $\mathrm{C}$ but they emphasize the importance of the resolution of the input parameters.

- time series of brightness temperature. Even if the grain size and density are assumed homogeneous the temperature profile is rarely uniform nor constant over time in snow and its variations can even be significant in the layer identified in the field based on the homogeneity of the grains and compactness. The variations of temperature near the surface are in general more rapid than the change of grain size and density due to metamorphism, and thus have the most important contribution to the short-term brightness temperature variations. Simulations at Dome $\mathrm{C}$ in Brucker et al. (2011a) show that even with grain size and density profiles taken constant over a few years (metamorphism is very slow at Dome $\mathrm{C}$ due to low temperature), the variations of brightness temperature are well reproduced $(\approx 2 \mathrm{~K})$ using measured time series of temperature profiles. Reducing the resolution of the temperature profile would result in a smoother time series.

\section{Validation of DMRT-ML with external data}

The comparison between measured brightness temperatures and results of DMRT-ML simulations using measured inputs was addressed in several studies: for a typical dry semiinfinite snowpack in Antarctica (Brucker et al., 2011a), for Arctic and sub-Arctic seasonal snowpacks (Roy et al., 2013) and for snow overlying ice as found in the ablation zone of the ice sheets (Dupont et al., 2012). In the three cases, it was necessary to estimate some parameters by optimization with respect to the measured brightness temperature. It is indeed difficult to measure all the input variables and parameters and the brightness temperatures with the same representativeness. In addition, some quantities - like the snow grain size and the soil properties - are notoriously difficult to determine in the field. The representation of snow grain in the DMRT-ML theory by spherical particles is also a conceptual difficulty.

Hence, the results of the comparisons and the errors estimated in these studies depends on the methodology and are meaningless out of the context of each study. Nevertheless, these studies converge on two facts. First, the grain size derived from specific surface area measurements, i.e., optical radius, needs to be increased by a factor between 2.8 and 3.5 to be suitable as input of DMRT-ML (see discussion in

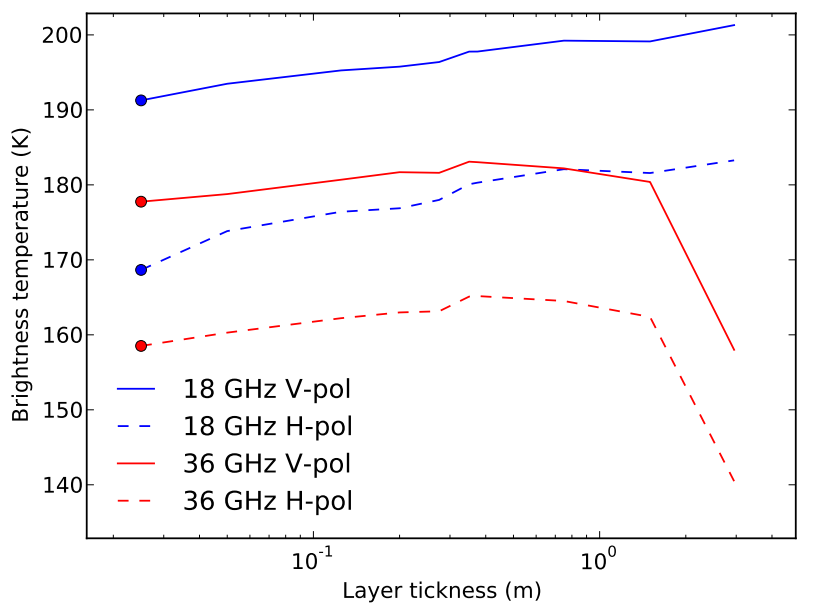

Fig. 10. Brightness temperature at 18.7 and $36.5 \mathrm{GHz}$ at Dome $\mathrm{C}$, Antarctica, simulated as a function of the resolution of the profiles of grain size and density. The original profiles are presented in Brucker et al. (2011a) and are composed of two parts: the upper $3 \mathrm{~m}$ were measured with a vertical resolution of $2.5 \mathrm{~cm}$ (simulation marked by a symbol) and the lower part ( 3 to $100 \mathrm{~m}$ depth) is a deterministic function of the depth. The profiles at lower resolutions are generated by merging successive layers in the upper part only to emphasize the influence of measured profile resolution, the lower part remaining unchanged. The $x$ axis reports the thickness of the layers, from $2.5 \mathrm{~cm}$ (original profile) to $3 \mathrm{~m}$ (i.e. one single layer for the upper part).

Roy et al., 2013). There is no evidence that similar adjustments would be required for the density or temperature measurements. Second, the model predicts reliable dependence between horizontal and vertical polarizations near the Brewster angle $\left(50-55^{\circ}\right)$. Figures 11 and 12 illustrate the latter point for the polarizations at 37 and $19 \mathrm{GHz}$ respectively. They show the brightness temperature at horizontal polarization versus vertical polarization for all the snow pits or pixels analyzed in Brucker et al. (2011a), Roy et al. (2013), and Dupont et al. (2012). Observations (in red) were acquired with a ground-based radiometer except at Dome C (stars), where measurements were recorded by the advanced microwave scanning radiometer for EOS (AMSR-E). DMRTML predictions are in blue. A large variety of environments are represented: Antarctica, ice-sheet ablation area, Arctic tundra, Arctic windy tundra, Arctic fen, and grassland. Each gray line links the observation and simulation result from the same snow pit or pixel. The length of each line illustrates the discrepancy between the observation and the simulation result of the order of $2-13 \mathrm{~K}$ but, as stated before, this is not an absolute error since it depends on the calibration procedure that differs between the studies. The relationship between the polarizations can be safely interpreted as it is almost independent of the calibration: Figs. 11 and 12 demonstrate that the measured brightness temperatures at both polarizations are highly correlated over a large range of about $120 \mathrm{~K}$ at 


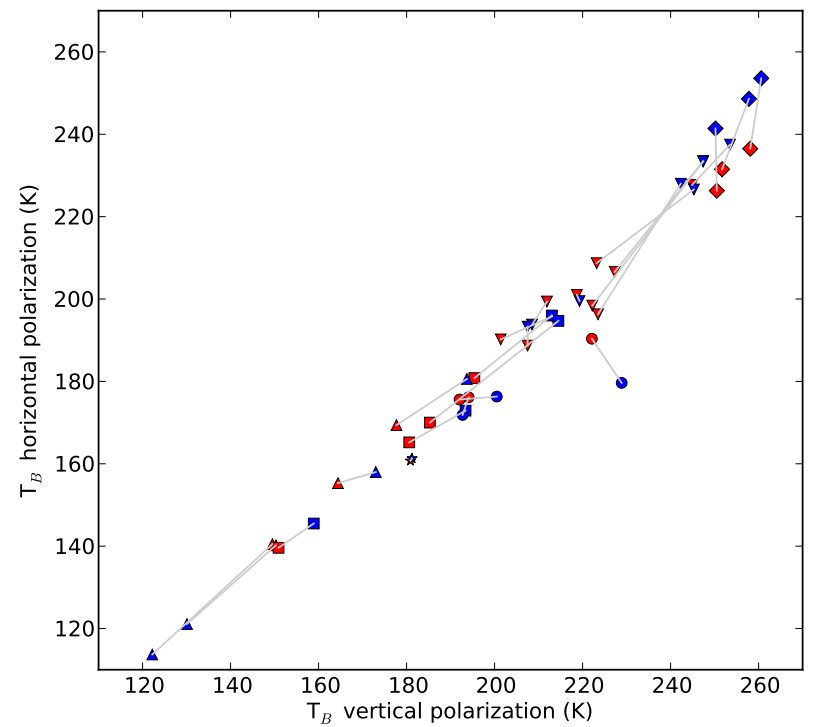

Fig. 11. Brightness temperatures at $55^{\circ}$ incidence angle and $37 \mathrm{GHz}$, at vertical polarization versus horizontal polarization for a variety of sites: Dome C, Antarctica (stars), Arctic tundra (triangles up), Arctic windy tundra (triangles down), Arctic fen (squares), grassland (diamonds), ice-sheet ablation or percolation areas (circles). Observations are in red and DMRT-ML predictions in blue. The gray lines link the observation and prediction of the same site.

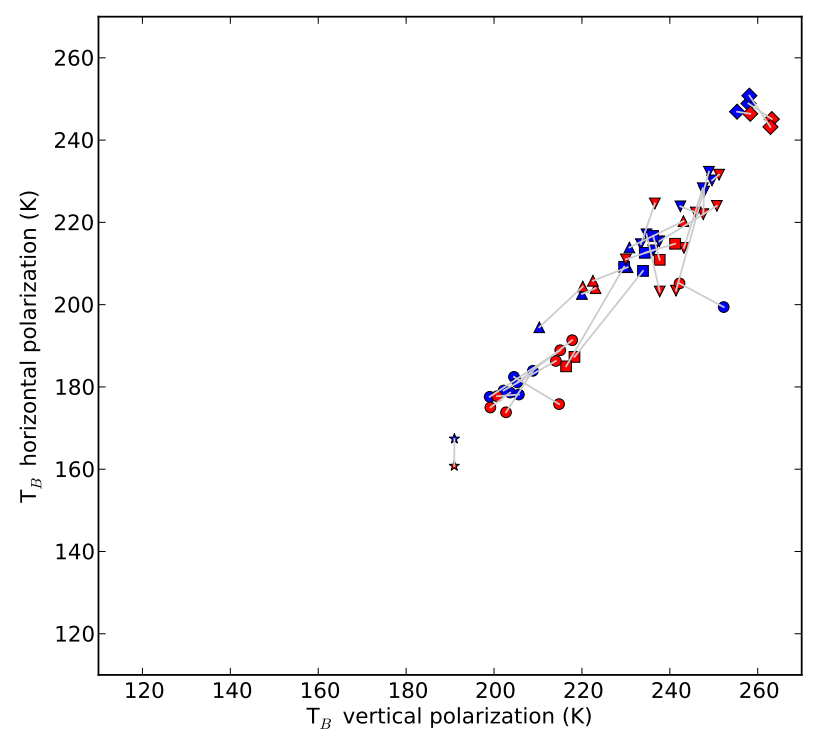

Fig. 12. Same as Fig. 11 for a frequency of $19 \mathrm{GHz}$.

$37 \mathrm{GHz}$ and $70 \mathrm{~K}$ at $19 \mathrm{GHz}$. Part of this correlation stems from the linear relationship between the brightness temperature and the snow physical temperature. However the range of physical temperature - typically from -55 to $0^{\circ} \mathrm{C}-$ is less than the brightness temperature ranges. It means that the emissivities at both polarizations are correlated. At $19 \mathrm{GHz}$, the correlation is lower than at $37 \mathrm{GHz}$. A probable explana- tion is the larger contribution of the substratum at the lower frequency due to the longer penetration depth. The important point is that the model nicely reproduces this general correlation. Even where the model and the observations disagree (i.e. long gray lines), the correlation between polarizations remains (i.e. the gray lines follow the general trend of the points).

\section{Conclusions}

The DMRT-ML is a physically based model used to compute brightness temperature at any frequency in the microwave range and at horizontal and vertical polarizations from input variables describing multi-layered snowpack and its environment. These variables and parameters include the profiles of snow temperature, density, grain size, stickiness, and liquid water content, the characteristics of the substratum (e.g. soil moisture, texture and temperature in the case of a soil substratum), and the downwelling atmospheric brightness temperature.

The paper presents the sensitivity of the microwave emission to the most important input variables and parameters and makes recommendations on the validity ranges of these variables and parameters, either constrained by the underlying DMRT-ML theory or by the specific DMRT-ML implementation. The validation of DMRT-ML with external in situ measurements is detailed in several studies (Brucker et al., 2011a; Roy et al., 2013; Dupont et al., 2012) for various environments. The error found between predicted and observed brightness temperatures ranged between 2 and $13 \mathrm{~K}$, which gives the magnitude of accessible errors but which depend on the methodology used for the comparison. In particular, the choice of the relationship to relate the measured grain size to the grain size metric relevant to the DMRT-ML theory is critical and no ideal solution exists yet. However, these studies add up to many others that have contributed to validate the DMRT-ML theory (Macelloni et al., 2001; Tsang and Kong, 2001; Tedesco et al., 2004; Grody, 2008). Currently, the most problematic point is probably the limited accuracy of the DMRT-ML theory for intermediate density values (about $300-500 \mathrm{~kg} \mathrm{~m}^{-3}$ ) that are commonly observed in natural snow and firn. An empirical correction of this problem has been recently proposed (Dierking et al., 2012). Even though it could be accurate enough, it has not the theoretical grounds that characterize the DMRT-ML theory and makes one of its merits. Further improvements are needed.

The main characteristics of the DMRT-ML implementation are the availability as an open source software, the efficiency of the computation due to the use of Fortran90 and LAPACK library, the wide range of cryospheric environments that can be modeled without any change of the source code (dry and wet snowpack, seasonal snow over soil, seasonal snow over bubbly ice, frozen lakes, perennial snow, etc.). In addition, modeling sea ice will be possible in the near 
future with the implementation of the dielectric constants of salted snow and water. These particularities are strong assets for the coupling of DMRT-ML with land surface models (Vionnet et al., 2012) and for the integration as an observation operator into data assimilation schemes or computationally intensive inverse methods. DMRT-ML is available for download at http://lgge.osug.fr/ picard/dmrtml/.

Acknowledgements. This work was supported by the French Programme National de Télédétection Spatiale, the Centre National d'Étude Spatiale, the Programme Internationale de Collaboration Scientifique (CNRS) between Grenoble's and Sherbrooke's universities and the Canadian Natural Sciences and Engineering Research Council. The manuscript was written in part during a visit at Sherbrooke university supported by the grant CMIRA 2012 ExploraPro, Region Rhone-Alpes. We thank S. Morin for helpful comments and the DMRT-ML user community for bug reports and suggestions through the DMRT-ML mailing-list.

Edited by: K. Gierens

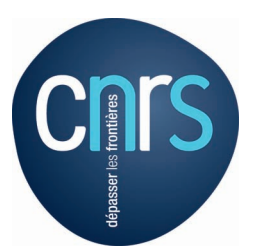

The publication of this article is financed by CNRS-INSU.

\section{References}

Abdalati, W. and Steffen, K.: Snowmelt on the Greenland Ice Sheet as Derived from Passive Microwave Satellite Data, J. Climate, 10, 165-175, 1997.

Abdalati, W. and Steffen, K.: Accumulation and hoar effects on microwave emission on the Greenland ice sheet dry snow zones, J. Glaciol., 44, 523-531, 1998.

Anderson, E., Bai, Z., Bischof, C., Blackford, S., Demmel, J., Dongarra, J., Du Croz, J., Greenbaum, A., Hammarling, S., McKenney, A., and Sorensen, D.: LAPACK Users' Guide, 3rd Edn., Soc. Industrial Appl. Math., Philadelphia, PA, 1999.

Arnaud, L., Picard, G., Champollion, N., Domine, F., Gallet, J., Lefebvre, E., Fily, M., and Barnola, J.: Measurement of vertical profiles of snow specific surface area with a $1 \mathrm{~cm}$ resolution using infrared reflectance: instrument description and validation, J. Glaciol., 57, 17-29, doi:10.3189/002214311795306664, 2011.

Arthern, R. J., Winebrenner, D. P., and Vaughan, D. G.: Antarctic snow accumulation mapped using polarization of $4.3-\mathrm{cm}$ wavelength microwave emission, J. Geophys. Res., 111, 10 pp., doi:10.1029/2004JD005667, 2006.

Borghese, F., Denti, P., and Saija, R.: Scattering from model nonspherical particles theory and applications to environmental physics, Springer, Berlin, New York, available at: http://search.ebscohost.com/login.aspx?direct=true\&scope= site $\& d b=$ nlebk\&db=nlabk\&AN=255898, 2007.

Brandt, R. E. and Warren, S. G.: Solar-heating rates and temperature profiles in Antarctic snow and ice, J. Glaciol., 39, 99-110, 1993.
Brogioni, M., Macelloni, G., Palchetti, E., Paloscia, S., Pampaloni, P., Pettinato, S., Santi, E., Cagnati, A., and Crepaz, A.: Monitoring Snow Characteristics With Ground-Based Multifrequency Microwave Radiometry, IEEE T. Geosci. Remote, 47, 36433655, doi:10.1109/TGRS.2009.2030791, 2009.

Brucker, L. and Markus, T.: Arctic-Scale Assessment of Satellite Passive Microwave Derived Snow Depth on Sea Ice using Operation IceBridge Airborne Data, J. Geophys. Res. Oceans, 118, 14 pp., doi:10.1002/jgrc.20228, 2013.

Brucker, L., Picard, G., and Fily, M.: Snow grain size profiles deduced from microwave snow emissivities in Antarctica, J. Glaciol., 56, 514-526, doi:10.3189/002214310792447806, 2010.

Brucker, L., Picard, G., Arnaud, L., Barnola, J., Schneebeli, M., Brunjail, H., Lefebvre, E., and Fily, M.: Modeling time series of microwave brightness temperature at Dome C, Antarctica, using vertically resolved snow temperature and microstructure measurements, J. Glaciol., 57, 171-182, 2011a.

Brucker, L., Royer, A., Picard, G., Langlois, A., and Fily, M.: Hourly simulations of the microwave brightness temperature of seasonal snow in Quebec, Canada, using a coupled snow evolution emission model, Remote Sens. Environ., 115, 1966-1977, doi:10.1016/j.rse.2011.03.019, 2011 b.

Butt, M. J. and Kelly, R. E. J.: Estimation of snow depth in the UK using the HUT snow emission model, Int. J. Remote Sens., 29, 4249-4267, doi:10.1080/01431160801891754, 2008.

Cavalieri, D., Markus, T., Ivanoff, A., Miller, J., Brucker, L., Sturm, M., Maslanik, J., Heinrichs, J., Gasiewski, A., Leuschen, C., Krabill, W., and Sonntag, J.: A Comparison of Snow Depth on Sea Ice Retrievals Using Airborne Altimeters and an AMSR-E Simulator, IEEE T. Geosci. Remote, 50, 3027-3040, doi:10.1109/TGRS.2011.2180535, 2012.

Champollion, N., Picard, G., Arnaud, L., Lefebvre, E., and Fily, M.: Hoar crystal development and disappearance at Dome C, Antarctica: observation by near-infrared photography and passive microwave satellite, The Cryosphere Discuss., 7, 175-217, doi:10.5194/tcd-7-175-2013, 2013.

Chandrasekhar, S.: Radiative transfer, New York: Dover, 1960.

Chang, W. and Tsang, L.: Conical electromagnetic waves diffraction from sastrugi type surfaces of layered snow dunes on Greenland ice sheets in passive microwave remote sensing, 122-125, IEEE, doi:10.1109/IGARSS.2011.6048913, 2011.

Chopra, K. L. and Reddy, G. B.: Optically selective coatings, Pramana, 27, 193-217, doi:10.1007/BF02846338, 1986.

Chuah, H. and Tan, H.: A Monte Carlo method for radar backscatter from a half-space random medium, IEEE T. Geosci. Remote, 27, 86-93, doi:10.1109/36.20278, 1989.

Colbeck, S. C.: The vapor diffusion coefficient for snow, Water Resourc. Res., 29, 109-116, doi:10.1029/92WR02301, 1993.

Derksen, C., Walker, A., and Goodison, B.: A comparison of 18 winter seasons of in situ and passive microwave-derived snow water equivalent estimates in Western Canada, Remote Sens. Environ., 88, 271-282, doi:10.1016/j.rse.2003.07.003, 2003.

Dierking, W., Linow, S., and Rack, W.: Toward a robust retrieval of snow accumulation over the Antarctic ice sheet using satellite radar, J. Geophys. Res., 117, D09110, doi:10.1029/2011JD017227, 2012.

Dobson, M., Ulaby, F., Hallikainen, M., and El-rayes, M.: Microwave Dielectric Behavior of Wet Soil-Part II: Dielectric 
Mixing Models, IEEE T. Geosci. Remote, GE-23, 35-46, doi:10.1109/TGRS.1985.289498, 1985.

Dupont, F., Picard, G., Royer, A., Fily, M., Roy, A., and Champollion, N.: Modeling the microwave emission of ice; Applications to the Cap Prud'Homme Blue Ice Area in Antarctica and to the Barnes Ice Cap superimposed ice in Arctic, IEEE Trans. Geo. Remote, in review, 2012.

Durand, M. and Margulis, S. A.: Correcting first-order errors in snow water equivalent estimates using a multifrequency, multiscale radiometric data assimilation scheme, J. Geophys. Res., 112, 15 pp., doi:10.1029/2006JD008067, 2007.

Durand, M., Kim, E. J., and Margulis, S. A.: Quantifying Uncertainty in Modeling Snow Microwave Radiance for a Mountain Snowpack at the Point-Scale, Including Stratigraphic Effects, IEEE T. Geosci. Remote, 46, 1753-1767, doi:10.1109/TGRS.2008.916221, 2008.

Flanner, M. G. and Zender, C. S.: Linking snowpack microphysics and albedo evolution, J. Geophys. Res., 111, 12 pp., doi:10.1029/2005JD006834, 2006.

Fung, A. K.: Microwave scattering and emission models and their applications, Remote sensing library, Artech House, Boston, USA, 1994.

Grody, N.: Relationship between snow parameters and microwave satellite measurements: Theory compared with Advanced Microwave Sounding Unit observations from 23 to $150 \mathrm{GHz}, \mathrm{J}$. Geophys. Res., 113, 17 pp., doi:10.1029/2007JD009685, 2008.

Grody, N. and Basist, A.: Global identification of snowcover using SSM/I measurements, IEEE T. Geosci. Remote, 34, 237-249, doi:10.1109/36.481908, 1996.

Gunn, G. E., Duguay, C. R., Derksen, C., Lemmetyinen, J., and Toose, P.: Evaluation of the HUT modified snow emission model over lake ice using airborne passive microwave measurements, Remote Sens. Environ., 115, 233-244, doi:10.1016/j.rse.2010.09.001, 2011.

Harlow, R. C. and Essery, R.: Tundra Snow Emissivities at MHS Frequencies: MEMLS Validation Using Airborne Microwave Data Measured During CLPX-II, IEEE T. Geosci. Remote, 50, 4262-4278, doi:10.1109/TGRS.2012.2193132, 2012.

Huang, C., Margulis, S. A., Durand, M. T., and Musselman, K. N.: Assessment of Snow Grain-Size Model and Stratigraphy Representation Impacts on Snow Radiance Assimilation: Forward Modeling Evaluation, IEEE T. Geosci. Remote, 50, 4551-4564, doi:10.1109/TGRS.2012.2192480, 2012.

Ishimaru, A.: Wave propagation and scattering in random media, IEEE Press; Oxford University Press, New York; Oxford; New York, 1997.

Ishimaru, A. and Kuga, Y.: Attenuation constant of a coherent field in a dense distribution of particles, J. Optical Soc. Am., 72, 1317, doi:10.1364/JOSA.72.001317, 1982.

Jiang, J. H. and Wu, D. L.: Ice and water permittivities for millimeter and sub-millimeter remote sensing applications, Atmos. Sci. Lett., 5, 146-151, doi:10.1002/as1.77, 2004.

Jin, Y. Q.: Electromagnetic scattering modelling for quantitative remote sensing, World Scientific, 1994.

Josberger, E. G. and Mognard, N. M.: A passive microwave snow depth algorithm with a proxy for snow metamorphism, Hydrol. Process., 16, 1557-1568, doi:10.1002/hyp.1020, 2002.

Kelly, R. E. J. and Chang, A. T. C.: Development of a passive microwave global snow depth retrieval algorithm for Special Sen- sor Microwave Imager (SSM/I) and Advanced Microwave Scanning Radiometer-EOS (AMSR-E) data, Radio Sci., 38, 41-1, doi:10.1029/2002RS002648, 2003.

Koenig, L. S., Steig, E. J., Winebrenner, D. P., and Shuman, C. A.: A link between microwave extinction length, firn thermal diffusivity, and accumulation rate in West Antarctica, J. Geophys. Res., 112, 10 pp., doi:10.1029/2006JF000716, 2007.

Kong, J. A., Shin, R., Shiue, J. C., and Tsang, L.: Theory and Experiment for Passive Microwave Remote Sensing of Snowpacks, J. Geophys. Res., 84, 5669-5673, doi:10.1029/JB084iB10p05669, 1979.

Kuipers Munneke, P., Picard, G., van den Broeke, M. R., Lenaerts, J. T. M., and van Meijgaard, E.: Insignificant change in Antarctic snowmelt volume since 1979, Geophys. Res. Lett., 39, 5 pp., doi:10.1029/2011GL050207, 2012.

Lacroix, P., Legresy, B., Remy, F., Blarel, F., Picard, G., and Brucker, L.: Rapid change of snow surface properties at Vostok, East Antarctica, revealed by altimetry and radiometry, Remote Sens. Environ., 113, 2633-2641, doi:10.1016/j.rse.2009.07.019, 2009.

Lemmetyinen, J., Pulliainen, J., Rees, A., Kontu, A., Qiu, Y., and Derksen, C.: Multiple-Layer Adaptation of HUT Snow Emission Model: Comparison With Experimental Data, IEEE T. Geosci. Remote, 48, 2781-2794, doi:10.1109/TGRS.2010.2041357, 2010.

Liang, D., Tse, K., Tan, Y., Tsang, L., and Ding, K. H.: Scattering and Emission in Snow Based on QCA/DMRT and Numerical Maxwell Model of 3-Dimentional Simulations (NMM3-D), IEEE MicroRad, In Proceedings of the IEEE 9th Specialist Meeting on Microwave Radiometry and Remote Sensing of the Environment (MicroRad 2006), 28 February-3 March 2006, San Juan, Puerto Rico, 197-202, doi:10.1109/MICRAD.2006.1677088, 2006.

Liang, D., Xu, X., Tsang, L., Andreadis, K. M., and Josberger, E. G.: The Effects of Layers in Dry Snow on Its Passive Microwave Emissions Using Dense Media Radiative Transfer Theory Based on the Quasicrystalline Approximation (QCA/DMRT), IEEE T. Geosci. Remote, 46, 3663-3671, doi:10.1109/TGRS.2008.922143, 2008.

Liang, D., Xu, P., Tsang, L., Gui, Z., and Chen, K.: Electromagnetic scattering by rough surfaces with large heights and slopes with applications to microwave remote sensing of rough surface over layered media, Prog. Electromagn. Res., 95, 199-218, doi:10.2528/PIER09071413, 2009.

Löwe, H. and van Herwijnen, A.: A Poisson shot noise model for micro-penetration of snow, Cold Reg. Sci. Technol., 70, 62-70, doi:10.1016/j.coldregions.2011.09.001, 2012.

Macelloni, G., Paloscia, S., Pampaloni, P., and Tedesco, M.: Microwave emission from dry snow: a comparison of experimental and model results, IEEE T. Geosci. Remote, 39, 2649-2656, doi:10.1109/36.974999, 2001.

Mätzler, C.: Improved Born approximation for scattering of radiation in a granular medium, J. Appl. Phys., 83, 6111-6117, 1998.

Mätzler, C.: Relation between grain-size and correlation length of snow, J. Glaciol., 48, 461-466, doi:10.3189/172756502781831287, 2002.

Mätzler, C. and Wegmüller, U.: Dielectric properties of freshwater ice at microwave frequencies, J. Phys. D Appl. Phys., 20, 1623, doi:10.1088/0022-3727/20/12/013, 1987. 
Mätzler, C. and Wiesmann, A.: Extension of the microwave emission model of layered snowpacks to coarse-grained snow, Remote Sens. Environ., 70, 317-325, 1999.

Mätzler, C., Aebischer, H., and Schanda, E.: Microwave dielectric properties of surface snow, IEEE J. Oceanic Eng., OE-9, 366-371, 1984.

Mätzler, C., Rosenkranz, P. W., Battaglia, A., and Wigneron, J. P.: Thermal microwave radiation - applications for remote sensing, no. 52 in IET, Electromagnetic Waves, London, UK, 2006.

Peake, W.: Interaction of electromagnetic waves with some natural surfaces, IRE Transactions on Antennas and Propagation, 7, 324-329, doi:10.1109/TAP.1959.1144736, 1959.

Picard, G. and Fily, M.: Surface melting observations in Antarctica by microwave radiometers: correcting 26-year time series from changes in acquisition hours, Remote Sens. Environ., 104, 325336, 2006.

Picard, G., Le Toan, T., Quegan, S., Caraglio, Y., and Castel, T.: Radiative transfer modeling of cross-polarized backscatter from a pine forest using the discrete ordinate and eigenvalue method, IEEE T. Geosci. Remote, 42, 1720-1730, doi:10.1109/TGRS.2004.831229, 2004

Picard, G., Brucker, L., Fily, M., Gallee, H., and Krinner, G.: Modeling timeseries of microwave brightness temperature in Antarctica, J. Glaciol., 55, 537-551, 2009.

Picard, G., Domine, F., Krinner, G., Arnaud, L., and Lefebvre, E.: Inhibition of the positive snow-albedo feedback by precipitation in interior Antarctica, Nature Climate Change, 2, 795-798, doi:10.1038/nclimate1590, 2012.

Pulliainen, J. T., Grandell, J., and Hallikainen, M. T.: HUT snow emission model and its applicability to snow water equivalent retrieval, IEEE T. Geosci. Remote., 37, 1378-1390, doi:10.1109/36.763302, 1999.

Rees, A., Lemmetyinen, J., Derksen, C., Pulliainen, J., and English, M.: Observed and modelled effects of ice lens formation on passive microwave brightness temperatures over snow covered tundra, Remote Sens. Environ., 114, 116-126, doi:10.1016/j.rse.2009.08.013, 2010.

Rosenfeld, S. and Grody, N.: Anomalous microwave spectra of snow cover observed from Special Sensor Microwave/Imager measurements, J. Geophys. Res., 105, 1491314926, doi:10.1029/1999JD900486, 2000.

Rosenkranz, P. W.: Water vapor microwave continuum absorption: A comparison of measurements and models, Radio Sci., 33, 919 928, doi:10.1029/98RS01182, 1998.

Roy, A., Picard, G., Royer, A., Montpetit, B., Dupont, F., Langlois, A., Derksen, C., and Champollion, N.: Brightness temperature simulations of the Canadian seasonal snowpack driven by measurements of the snow specific surface area, , IEEE T. Geosci. Remote, 1-13, doi:10.1109/TGRS.2012.2235842, 2013.

Saunders, R., Matricardi, M., and Brunel, P.: An improved fast radiative transfer model for assimilation of satellite radiance observations, Q. J. Roy. Meteor. Soc., 125, 1407-1425, doi:10.1256/smsqj.55614, 1999.

Schneider, D. P.: Spatial and temporal variability of Antarctic ice sheet microwave brightness temperatures, Geophys. Res. Lett., 29, 1964, doi:10.1029/2002GL015490, 2002.

Schneider, D. P., Steig, E. J., and Comiso, J. C.: Recent climate variability in Antarctica from satellite-derived temperature data, J. Climate, 17, 1569-1583, 2004.
Shih, S., Ding, K., Kong, J. A., Yang, Y. E., Davis, R. E., Hardy, J. P., and Jordan, R.: Modeling of Millimeter Wave Backscatter of Time-Varying Snowcover, Prog. Electromagn. Res., 16, 305330, doi:10.2528/PIER97012600, 1997.

Shuman, C. A., Alley, R. B., Anandakrishnan, S., and Stearns, C. R.: An empirical technique for estimating near-surface air temperature trends in central Greenland from SSM/I brightness temperatures, Remote Sens. Environ., 51, 245-252, 1995.

Stamnes, K., Tsay, S. C., Jayaweera, K., and Wiscombe, W.: Numerically stable algorithm for discrete-ordinate-method radiative transfer in multiple scattering and emitting layered media, Appl. Optics, 27, 2502-2509, 1988.

Stogryn, A.: A Study of the Microwave Brightness Temperature of Snow from the Point of View of Strong Fluctuation Theory, IEEE T. Geosci. Remote, 24, 220-231, doi:10.1109/TGRS.1986.289641, 1986.

Surdyk, S. and Fily, M.: Results of a stratified snow emissivity model based on the wave approach: application to the Antarctic ice sheet, J. Geophys. Res., 100, 8837-8848, 1995.

Takala, M., Luojus, K., Pulliainen, J., Derksen, C., Lemmetyinen, J., Kärnä, J., Koskinen, J., and Bojkov, B.: Estimating northern hemisphere snow water equivalent for climate research through assimilation of space-borne radiometer data and groundbased measurements, Remote Sens. Environ., 115, 3517-3529, doi:10.1016/j.rse.2011.08.014, 2011.

Tedesco, M. and Kim, E. J.: Intercomparison of Electromagnetic Models for Passive Microwave Remote Sensing of Snow, IEEE T. Geosci. Remote, 44, 2654-2666, doi:10.1109/TGRS.2006.873182, 2006.

Tedesco, M., Kim, E., Cline, D., Graf, T., Koike, T., Hardy, J., Armstrong, R., and Brodzik, M.: The cold land processes experiment (CLPX-1): analysis and modelling of LSOS data (IOP3 period), 6, 3725-3728, IEEE, doi:10.1109/IGARSS.2004.1369931, 2004.

Tedesco, M., Abdalati, W., and Zwally, H. J.: Persistent surface snowmelt over Antarctica (1987-2006) from 19.35 $\mathrm{GHz}$ brightness temperatures, Geophys. Res. Lett., 34, 6 pp., doi:10.1029/2007GL031199, 2007.

Toure, A. M., Goita, K., Royer, A., Kim, E. J., Durand, M., Margulis, S. A., and Lu, H.: A Case Study of Using a Multilayered Thermodynamical Snow Model for Radiance Assimilation, IEEE T. Geosci. Remote, 49, 2828-2837, doi:10.1109/TGRS.2011.2118761, 2011.

Tsang, L. and Kong, J. A.: Scattering of electromagnetic waves, 3, Advanced Topics, Wiley Interscience, 2001.

Tsang, L., Kong, J. A., and Shin, R. T.: Theory of Microwave Remote Sensing, Wiley-Interscience, New York, 1985.

Tsang, L., Chen, C. T., Chang, A. T. C., Guo, J., and Ding, K. H.: Dense Media Radiative Transfer Theory Based on Quasicrystalline Approximation with Application to Passive Microwave Remote Sensing of Snow, Radio Sci., 35, 741-49, 2000a.

Tsang, L., Kong, J. A., Ding, K. H., and Ao, C.: Scattering of electromagnetic waves, 2, numerical solutions, Wiley Interscience, New York, 705 pp., http://www.amazon.com/ Scattering-Electromagnetic-Waves-Numerical-Simulations/dp/ 0471388009/ref=pd_sim_b_1, 2000b.

Tsang, L., Pan, J., Liang, D., Li, Z. X., Cline, D., and Tan, Y. H.: Modeling active microwave remote sensing of snow using dense media radiative transfer(DMRT) theory with multiple scattering 
effects, IEEE T. Geosci. Remote, 45, 990-1004, 2007.

Tsang, L., Xu, P., and Chen, K. S.: Third and fourth stokes parameters in polarimetric passive microwave remote sensing of rough surfaces over layered media, Microw. Opt. Techn. Lett., 50, 3063-3069, 2008.

Twomey, S. and Bohren, C. F.: Simple Approximations for Calculations of Absorption in Clouds, J. Atmos. Sci., 37, 2086-2095, doi:10.1175/1520-0469(1980)037<2086:SAFCOA > 2.0.CO;2, 1980.

Ulaby, F. T., Moore, R. K., and Fung, A. K.: Microwave Remote Sensing, 3, Artech house, Norwood, MA, 1986.

Vaughan, D. G., Bamber, J. L., Giovinetto, M., Russell, J., and Cooper, A. P. R.: Reassessment of net surface mass balance in Antarctica, J. Climate, 12, 933-946, 1999.

Vionnet, V., Brun, E., Morin, S., Boone, A., Faroux, S., Le Moigne, P., Martin, E., and Willemet, J.-M.: The detailed snowpack scheme Crocus and its implementation in SURFEX v7.2, Geosci. Model Dev., 5, 773-791, doi:10.5194/gmd-5-773-2012, 2012.

Warren, S. G.: Optical Properties of Snow (Paper 1R1505), Rev. Geophys. Space Phys., 20, 67-89, 1982.

Watanabe, O.: Distribution of surface features of snow cover in Mizuho Plateau, Tech. rep., 1978.
West, R., Tsang, L., and Winebrenner, D. P.: Dense Medium Radiative Transfer theory for two scattering layers with a Rayleigh distribution of particle sizes, IEEE T. Geosci. Remote, 31, 436437, 1993.

West, R. D., Winebrenner, D. P., Tsang, L., and Rott, H.: Microwave emission from density-stratified Antarctic firn at $6 \mathrm{~cm}$ wavelength, J. Glaciol., 42, 63-76, 1996.

Wiesmann, A. and Mätzler, C.: Microwave emission model of layered snowpacks, Remote Sens. Environ., 70, 307-316, 1999.

Wiesmann, A., Mätzler, C., and Weise, T.: Radiometric and structural mesurements of snow samples, Radio Sci., 33, 273-289, 1998.

Winebrenner, D. P., Arthern, R. J., and Shuman, C. A.: Mapping Greenland accumulation rates using observations of thermal emission at 4.5-cm wavelength, J. Geophys. Res., 106, 3391933934, doi:10.1029/2001JD900235, 2001.

Zwally, H. J.: Microwave emissivity and accumulation rate of polar firn, J. Glaciol., 18, 195-214, 1977. 